\title{
Structural Characterization of Self-Assemblies of
}

\author{
New Omega-3 Lipids: Docosahexaenoic Acid and
}

\section{Docosapentaenoic Acid Monoglycerides}

\author{
Xianrong Shao ${ }^{1}$, Gizem Bor ${ }^{1, \Perp}$, Sabah Al-Hosayni ${ }^{1, \Perp}$, Stefan Salentinig ${ }^{2}$, Anan \\ Yaghmur $^{1, *}$ \\ ${ }^{1}$ Department of Pharmacy, Faculty of Health and Medical Sciences, University of Copenhagen, \\ Universitetsparken 2, DK-2100 Copenhagen Ø, Denmark \\ ${ }^{2}$ Laboratory for Biointerfaces, Empa, Swiss Federal Laboratories for Materials Science and \\ Technology, Lerchenfeldstrasse 5, 9014 St. Gallen, Switzerland
}

"Contributed equally.

"Corresponding Author: e-mail address: anan.yaghmur@sund.ku.dk

This document is the accepted manuscript version of the following article:

Shao, X., Bor, G., Al-Hosayni, S., Salentinig, S., \& Yaghmur, A. (2018). Structural characterization of self-assemblies of new omega-3 Lipids: Docosahexaenoic Acid and Docosapentaenoic Acid Monoglycerides. Physical Chemistry Chemical Physics. https:// doi.org/10.1039/C8CP04256J 


\section{ABSTRACT:}

The attractiveness of new omega-3 ( $\omega-3)$ polyunsaturated fatty acid (PUFA) monoglycerides (MAGs) lies in the amphiphilic nature, and the beneficial health effects as PUFA precursors in various disorders including cancer, pulmonary hypertension, and inflammatory diseases. For exploring the potential therapeutic applications of these new amphiphilic lipids, particularly as main lipid constituents in the development of nanocarriers for delivery of drugs and PUFAs, it is of paramount importance to gain insight into their self-assembly behavior on exposure to excess water. This work describes the structural characteristics of self-assemblies based on two newly synthesized MAGs, namely docosahexaenoic acid (MAG-DHA) and docosapentaenoic acid (MAG-DPA) monoglycerides on exposure to excess water. We found that both lipids tend to form dominant inverse hexagonal $\left(\mathrm{H}_{2}\right)$ phase in excess water at $25{ }^{\circ} \mathrm{C}$ and a temperature-triggered structural transition to an inverse micellar solution $\left(\mathrm{L}_{2}\right.$ phase) is detected similar to that recently reported (A. Yaghmur et al., Langmuir, 2017, 33, 14045-14057) for eicosapentaenoic acid monoglyceride (MAG-EPA). An experimental SAXS structural evaluation study on the temperature-dependent behavior of these new monoglycerides is provided, and the effects of the unsaturation degree and the fatty acyl chain length on the self-assembled structural features in excess water and on the $\mathrm{H}_{2}-\mathrm{L}_{2}$ phase transition temperature is discussed. In addition, hexosomes stabilized by using the triblock copolymer F127 and the food-grade emulsifier citrem were investigated to gain insights into the effects of stabilizer and temperature on the internal nanostructure. These nanoparticles are attractive for use in the development of nanocarriers for delivering drugs and/or nutritional compounds as the beneficial health effects of $\omega-3$ PUFA monoglycerides can be combined with loaded therapeutic agents or nutraceuticals. 


\section{INTRODUCTION}

The new family of omega-3 ( $\omega-3)$ polyunsaturated fatty acid (PUFA) monoglycerides (MAGs) of docosahexaenoic acid (DHA; 22:6, n-3), docosapentaenoic acid (DPA; 22-5, n-3), and eicosapentaenoic acid (EPA; 20:5, n-3) hold potential in the development of carriers for the delivery of drugs and functional foods owning to their unique structural properties and health-promoting effects $^{1-11}$. Similar to their corresponding long-chain omega-3 $(\omega-3)$ polyunsaturated fatty acids (PUFAs) that are typically present in fish oil extracts, particularly EPA and DHA with recommended daily intake ${ }^{12}$ of $200-500 \mathrm{mg}$, these $\omega-3$ amphiphilic lipids have beneficial health effects in various disorders including inflammation ${ }^{1,2,4,5}$, cardiovascular disease (CVD) events ${ }^{2,4-6}$, asthma $^{2,7-10}$, allergic asthma $a^{2,4,10}$, hypertension ${ }^{5}$, and cancer ${ }^{1,3,11}$. They can also be used as cancer ${\text { chemo-preventive } \text { agents }^{8,9,11} \text {, and radio-enhancers }}^{8,9,11}$ for radiotherapy of cancer $^{8,9}$. The accumulating evidences on their role in modulating inflammatory events, and their anti-proliferative and pro-apoptotic effects may have implications for the treatment of inflammatory and cancer diseases ${ }^{1,7,13,14}$. It was also reported that MAG-DHA, MAG-DPA, and MAG-EPA are safe precursors that are absorbed by the gastrointestinal (GI) $\operatorname{tract}^{10,15}$, then metabolized and hydrolyzed to corresponding DHA, DPA and EPA derivatives including resolvins and protectins ${ }^{1,7,13,14}$. These derivatives can be found in blood circulation or other body tissues ${ }^{6,8,10}$ and play an important role in mediating pro-inflammatory processes, cell proliferative, and invasion and immune-modulatory effects in numerous diseases ${ }^{1,7,13,14}$.

The synthesis of MAG-DHA, MAG-DPA, and MAG-EPA is carried out through esterification at the $s n-1$ position of the glycerol backbone by using as starting materials the following highly purified corresponding ethyl esters: ethyl docosahexaenoate, ethyl docasapentaenoate, and ethyl eicosapentaenoate, respectively ${ }^{2,8,10}$. In addition to these monoglycerides, $\omega-3$ PUFAs are also 
commercially available in different forms. For instance, there are phospholipid-enriched formulations, and supplements based on free fatty acids (FFAs), ethyl esters (EEs) and triacylglycerides (TAGs). It was reported that $\omega-3$ monoglycerides have a higher bioavailability as compared to ethyl esters and TAGs ${ }^{2,3,6,16}$.

Another interesting aspect that makes $\omega-3$ PUFA monoglycerides superior to other $\omega-3$ PUFA forms is their amphiphilicity rendering them attractive as main lipid constituents for the formation of self-assemblies with possible drug and functional delivery implications. Owning to the reported health beneficial effects, the use of these new single amphiphilic lipids in the development of drug and $\omega-3$ PUFA delivery systems may prove to be an advantageous and simple approach as compared to counterparts based on binary, ternary, or multi-component lipid mixtures ${ }^{17,18}$ containing the corresponding $\omega$-3 PUFAs. Among these counterparts, recent studies reported on the formation and characterization of lamellar and non-lamellar lipid crystalline nanoparticles based on the binary lipid mixtures of EPA and DHA with monoolein (MO; C18:1) and phytantriol (PHYT), respectively $^{17,18}$.

We recently reported on the temperature-dependence of binary MAG-EPA/water system under limited and fully hydrated conditions ${ }^{19}$. We found that MAG-EPA tends to form a dominant inverse hexagonal $\left(\mathrm{H}_{2}\right)$ phase in excess water and a transition to an inverse micellar solution $\left(\mathrm{L}_{2}\right.$ phase $)$ is detected at approximately $56{ }^{\circ} \mathrm{C}$. In addition, we showed that the fragmentation of the fully hydrated $\mathrm{H}_{2}$ phase in the presence of the co-polymeric stabilizer F127 or the food-grade emulsifier citrem leads to the formation of colloidally stable hexosomes. It is interesting that it was possible to prepare F127-stablized MAG-EPA hexosomes suitable for loading temperature-sensitive and solvent-labile drugs by using a low-energy emulsification. As a continuation, our present work focuses on investigating the influence of temperature on the structural characterization of both dispersed and non-dispersed bulk phases based on MAG-DHA and MAG-DPA by using 
synchrotron small-angle X-ray scattering (SAXS). In addition, cryogenic transmission electron microscopy (cryo-TEM), nanoparticle tracking analysis (NTA), and zeta potential measurements were performed to gain insight into the morphological features, the size characteristics, and the surface charge of the produced nanoparticles. It is also of interest to explore the effects of unsaturation degree and fatty acyl chain length on the structural features of these two new $\omega-3$ PUFA amphiphilic lipids as compared to MAG-EPA, and two monounsaturated monoglycerides: mono-10(Z)-heptadecenion (MHO, C17:1), and MO (Figure 1). The latter is the most investigated monounsaturated monoglycerides in the preparation of inverse non-lamellar liquid crystalline phases and their corresponding nanoparticles (mainly cubosomes and hexosomes) ${ }^{18,21-28}$.

In recent years, there is a growing interest in the use of non-lamellar liquid crystalline nanoparticles (mainly cubosomes and hexosomes) as nanocarriers for delivering various drugs and peptides, as well as for bio-imaging applications ${ }^{20-27}$. Taken into consideration the beneficial health and therapeutic effects of $\omega-3$ PUFA monoglycerides, and their use as main lipid constituents in the production of non-lamellar liquid crystalline nanoparticles, this family of new lipids have the potential to be used in the development of nanocarriers for combined delivery of drugs and $\omega-3$ PUFAs.

\section{EXPERIMENTAL SECTION}

\subsection{Materials}

Docosahexaenoic acid monoglyceride (MAG-DHA) with purity of $>99 \%$ was purchased from NUCHEK PREP, INC. (MN, USA). Docosapentaenoic acid monoglyceride (MAG-DPA) with purity of $>99 \%$, mono-10(Z)-heptadecenoin (monoheptadecenoin, $\mathrm{MHO}$ ) with purity of $>99 \%$, and 
monoolein (MO) with purity of $>99 \%$ were purchased from Larodan AB (Solna, Sweden). Pluronic

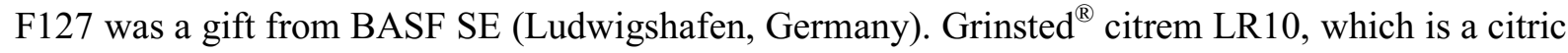
acid ester of monoglycerides and diglycerides made from sunflower oil, was received as a gift from Danisco A/S (Copenhagen, Denmark). All ingredients were used without further purification. The water was double distilled.

2.2 Preparation of Non-Dispersed Binary lipid/Water Systems. The samples were prepared as described for the preparation of hydrated MAG-EPA samples in our recent report ${ }^{19}$. MAG-DHA, MAG-DPA, mono-10(Z)-heptadecenoin (MHO), and monoolein (MO) were hydrated by adding dropwise MQ water and carrying out 5 freeze-thaw cycles between liquid nitrogen and room temperature and homogenizing during the thawing steps by vigorous vortex. The vials of the prepared samples at different water content were flushed with nitrogen gas, capped, covered by aluminum foil, and then incubated at $4{ }^{\circ} \mathrm{C}$ for at least one week before performing the SAXS measurements. The fully hydrated samples were formed at water concentration of $40 \mathrm{wt} \%$ for MAG-DHA and $90 \mathrm{wt} \%$ for MAG-DPA; whereas the two MAG-DHA samples prepared under limited hydration condition contained 15 and $25 \mathrm{wt} \%$ water.

2.3 Preparation of Aqueous MAG-DHA and MAG-DPA Nanodispersions. As described in our recent report ${ }^{19}$ on the formation of MAG-EPA nanoparticles, the aqueous nanodispersions based on MAG-DHA and MAG-DPA were prepared in the presence of the polymeric stabilizer F127 or the negatively charged food-grade emulsifier citrem. F127-stabilized nanodispersion $\left(\mathrm{D}_{\mathrm{F} 127}\right)$ contained $5 \mathrm{wt} \%$ MAG-DHA (or MAG-DPA), $1 \mathrm{wt} \% \mathrm{~F} 127$, and $94 \mathrm{wt} \%$ water. The nanodispersions based on the binary citrem/MAG-DHA mixtures containing $5 \mathrm{wt} \%$ citrem/MAG-DHA mixture and $95 \mathrm{wt} \%$ 
water were prepared at two different citrem/MAG-DHA weight ratios of 2:3 (sample CM2:3) and 3:2 (sample CM3:2). The applied high-energy emulsification method for the production of F127and citrem-stabilized nanodispersions was as following: MAG-DHA, MAG-DPA, and the two binary citrem/MAG-DHA mixtures were dispersed in excess water by means of ultrasonication (Qsonica MIS 4417 and Qsonica MIS 4659 4-tip horn (Qsonica LLC., Newtown, CT, USA), for 2 min in pulse mode ( $5 \mathrm{~s}$ pulses interrupted by $2 \mathrm{~s}$ breaks) at $25 \%$ of maximum power until stable milky solutions were obtained. The vials of all prepared samples were flushed with nitrogen gas, capped, covered by aluminum foil, and then incubated at room temperature before performing the SAXS experiments. It is worth noting that citrem was tested as stabilizer for MAG-DPA nanodispersions at the mentioned citrem/MAG-DPA ratios of $2: 3$ and $3: 2$ but the produced nanodispersions were not colloidally stable and therefore excluded from the present study.

2.4 Synchrotron Small Angle X-ray Scattering (SAXS). SAXS patterns were recorded at the Austrian SAXS beamline in the synchrotron light source ELETTRA (Trieste, Italy). The 2D SAXS patterns were acquired using a Pilatus3 1M detector (Dectris Ltd, Baden, Switzerland; active area $169 \times 179 \mathrm{~mm}^{2}$ with a pixel size of $172 \mu \mathrm{m}$ ) and integrated into one-dimensional (1-D) scattering function I $(q)$ using Fit2D and then analyzed with Igor Pro (Wavemetrics, Inc., Lake Oswego, OR). An X-ray beam having wavelength of $1.54 \AA$ at an $\mathrm{X}$-ray energy of $8 \mathrm{keV}$ was used. The sample to detector distance was $1314 \mathrm{~mm}$ covering a $q$-range of interest from about 0.07 to $5.0 \mathrm{~nm}^{-1}(q=4 \pi \sin \theta / \lambda$, where $\lambda$ is the wavelength and $2 \theta$ is the scattering angle). Silver behenate $\left(\mathrm{CH}_{3}-\left(\mathrm{CH}_{2}\right)_{20}-\mathrm{COOAg}\right.$ with a $d$-spacing value of $58.38 \AA$ ) was used as a standard to calibrate the angular scale of the measured intensity. In our investigations, $1 \mathrm{~mm}$ diameter quartz capillaries were used and the samples were thermostated with a water bath (temperature stability $\pm 0.1{ }^{\circ} \mathrm{C}$, Unistat $\mathrm{CC}$, Huber, Offenburg, Germany). 
Before carrying out the continuous temperature scan experiments, static measurements were performed at 25 or $37^{\circ} \mathrm{C}$ with an exposure time of $20 \mathrm{~s}$ per frame with $3 \mathrm{~s}$ delay between 5 frames. The temperature ramps in the range of $5-60{ }^{\circ} \mathrm{C}$ were programmed with scan rate of 1 ${ }^{\circ} \mathrm{C} / \mathrm{min}$ typically recording an X-ray pattern every $1{ }^{\circ} \mathrm{C}$. After the raw data had been corrected for detector efficiency and the background scattering, all Bragg peaks were fitted by Lorentzian distributions. The lattice parameters of the inverted-type bicontinuous cubic $\left(\mathrm{Q}_{2}\right)$ and discontinuous hexagonal $\left(\mathrm{H}_{2}\right)$ phases were derived from the SAXS diffraction patterns. We note that in each respective phase regime only the strongest reflections were considered for calculating the corresponding lattice parameter. For the inverse micelles solution (the $\mathrm{L}_{2}$ phase), the characteristic distance, $d=2 \pi / q$, was calculated.

2.5 Cryo-transmission electron microscopy (Cryo-TEM). The morphological characterization of MAG-DHA and MAG-DPA nanoparticles was done as previously described ${ }^{28}$. Briefly, 3-4 $\mu \mathrm{L}$ of the nanodispersions were applied on a hydrophilized lacey carbon 300 mesh copper grid (Ted Pella Inc., California, USA). The excess sample on the grid was then blotted with filter paper at blotting time $5 \mathrm{~s}$, blotting force 0 , temperature $4{ }^{\circ} \mathrm{C}$ and $100 \%$ humidity (FEI Vitrobot IV, Holland), and was rapidly plunged into liquid-nitrogen cooled ethane $\left(-180^{\circ} \mathrm{C}\right)$. The samples' observations were done with Tecnai G2 20 transmission electron microscope (FEI, Holland) at a voltage of $200 \mathrm{kV}$ under low-dose rate $\left(\sim 5 \mathrm{e} / \AA^{2} \mathrm{~s}\right)$. Images were then recorded using a FEI Eagle camera $4 \times 4 \mathrm{k}$ at a nominal magnification of $69,000 \mathrm{x}$ resulting in a final image sampling of $0.22 \mathrm{~nm} /$ pixel.

2.6 Zeta Potential. The zeta potential of the nanodispersions was measured using a Zetasizer Nano ZS (Malvern Instruments, Worcestershire, U.K.) equipped with a $633 \mathrm{~nm}$ laser and $173^{\circ}$ detection optics. Measurements were performed at room temperature on samples diluted 100x in water. 
Malvern DTS v. 6.34 software (Malvern instruments, Worcestershire, U.K.) was used for data acquisition and analysis. For the viscosity and refractive index, the values of MQ water were used.

2.7 Nanoparticle tracking analysis (NTA). Size characterization of the nanodispersions was conducted at room temperature using Nanoparticle Tracking Analysis (NTA) on NanoSight NS300 (Malvern Instruments Ltd, Worcestershire, UK) mounted with a $405 \mathrm{~nm}$ laser. Prior to measurements, the samples were diluted $10^{4}$ times in MQ water $(18.2 \mathrm{M} \Omega \cdot \mathrm{cm})$ to reach a measured concentration between $10^{8}$ and $10^{9}$ particles $/ \mathrm{mL}$. All nanodispersions were measured in triplicate using same camera settings and analysed under the same criteria to allow direct comparison. All measurements were based on 2000 individually detected nanoparticles from 5 to 9 videos from different locations in the sample. The recorded videos were analysed using Malvern software (NTA 3.2 Dev Build 3.2.16). Deionized water was measured as a control at identical instrumental settings.

\section{RESULTS AND DISCUSSION}

\subsection{Effects of Unsaturation Degree and Fatty Acyl Chain Length on the Structural Features.}

A set of synchrotron SAXS experiments were performed at $25^{\circ} \mathrm{C}$ to gain insight into the effects of the unsaturation degree and the fatty acyl chain length of the new $\omega-3$ monoglycerides MAG-DPA and MAG-DHA on the structural properties of their bulk non-dispersed fully hydrated selfassemblies as compared to those based on monoolein (MO), mono-10(Z)-heptadecenoin (MHO), and MAG-EPA ${ }^{19}$ respectively (Figure 2A). For the monounsaturated monoglycerides MO (a C18:1 monoglyceride) and MHO (a C17:1 monoglyceride) in excess water, at least seven characteristic reflections were detected for an inverse bicontinuous cubic phase of the symmetry Pn3m with 
lattice parameter of about 10.00 and $12.17 \mathrm{~nm}$, respectively. These results are consistent with previous reports $^{29,30}$ on the tendency of these lipids to form inverse bicontinuous cubic Pn3m phase in excess water. It is interesting that the fully hydrated MHO has a greater lattice parameter (almost $2 \mathrm{~nm}$ larger) than that of the fully hydrated MO. At the molecular level, MO (oleic acid-containing lipid) has an 18-carbon fatty acyl chain length with one cis double bond at the position 9 (C9) of its carbon atom backbone; whereas its counterpart MHO (cis-10-heptadecenoic acid-containing lipid) has one carbon atom less on its fatty acyl chain and the cis double bond is shifted to position 10 (C10). Thus, the SAXS results suggest that the lattice parameter of the cubic Pn $3 m$ phase in excess water is very sensitive to changes in the fatty acyl chain length and/or the location of the double bond at the carbon atom backbone. It was reported for monounsaturated monoglycerides having propensity for inverse bicontinuous cubic $\operatorname{Pn} 3 m$ phase formation in excess water that the fatty acyl chain length and the double bond position have a significant influence on the hydrophilic nanochannels of the cubic phase ${ }^{31}$. It was found that shortening the fatty acyl chain length leads to an enlargement of the hydrophilic nanochannels of the cubic Pn3m phase ${ }^{31}$. The relocation of the double bond closer to the methyl end of the fatty acyl chain of MHO by one methylene group and away from its hydrophilic headgroup as compared to MO could also affect the structural properties of the cubic Pn3m phase due to possible alteration in the shape of this lipid at the molecular level. In this context, it was reported that the relocation of the double bond closer to the methyl end of the fatty acyl chain by two methylene group in monovaccenin (a C18:1 monoglyceride with a double position at the position $\mathrm{C} 11$ ), as compared to $\mathrm{MO}$ is associated with a slight enlargement of the hydrophilic nanochannels of the inverse cubic Pn3m phase in excess water ${ }^{31,32}$. To understand the reason for such structural change, Caffery and co-workers ${ }^{31}$ followed a basic approach based on the critical packing effect (CPP), known also as the molecular wedge shape factor, which is defined as ${ }^{33}$ : 


$$
C P P=v_{s} / a_{0} l
$$

where $v_{s}$ is the hydrophobic chain volume, $a_{0}$ is the headgroup area, and $l$ is the hydrophobic chain length. Both MO and monovaccenin tend to form inverse non-lamellar liquid crystalline phases in excess water at ambient temperatures, and therefore, the CPP is $>1$. However, it was proposed that the movement of the double bond far away from the hydrophilic headgroup in monovaccenin leads to a generation of a "slimmer" amphiphilic compound with smaller CPP than MO leading to a slight enlargement of the hydrophilic nanochannels of the inverse cubic Pn3m phase. For MHO, it seems the combined effects of decreasing slightly the chain length and the movement of the double bond on the carbon atom backbone generate even a more "slimmer" amphiphilic compound than monovaccenin leading to a significant enlargement of the hydrophilic nanochannels of the inverse cubic $P n 3 m$ phase as shown above.

Returning to the new $\omega-3$ PUFA monoglycerides (MAG-DHA, MAG-EPA and MAG-DPA) that have same hydrophilic headgroup and ester linkage at position $\mathrm{C} 1$ between the glycerol backbone and the corresponding PUFAs, it was our aim to shed light on the effects of the PUFA chain length and unsaturation degree on their self-assemblies in excess water. In our recent report ${ }^{19}$, we found that MAG-EPA (a monoglyceride of C20:5 n-3) exhibits at $25{ }^{\circ} \mathrm{C}$ an inverse hexagonal $\left(\mathrm{H}_{2}\right)$ phase with a lattice parameter of about $4.94 \mathrm{~nm}$ in excess water (Figure 2A). The SAXS patterns for the counterparts MAG-DHA (a monoglyceride of C22:6 n-3) and MAG-DPA (a monoglyceride of $\mathrm{C} 22: 5$ n-3) in excess water at $25{ }^{\circ} \mathrm{C}$ (Figure 2A) indicate also the formation of $\mathrm{H}_{2}$ phases with lattice parameters of 4.81 and $5.00 \mathrm{~nm}$, respectively.

It has been reported that monounsaturated monoglycerides of cis-fatty acids with relatively shorter acyl chains than MO such as monomyristolein ${ }^{34,35}$ (a C14:9 monoglyceride) tend to form lamellar 
$\left(\mathrm{L}_{\alpha}\right)$ liquid crystalline phases on expose to excess water at room temperature. Increasing the fatty acyl chain length results in more wedge-shaped monoglycerides and enhances therefore the propensity for the formation of inverse lyotropic non-lamellar phases (an inverse bicontinuous cubic Pn3m phase or a discontinuous hexagonal $\left(\mathrm{H}_{2}\right)$ phase) in excess water ${ }^{29-32,35-39}$. As compared to MO and MHO, $\omega-3$ PUFA monoglycerides are more wedged-shaped compounds and have more highly curved lipid-water interfaces leading to the preferential formation of $\mathrm{H}_{2}$ phase. There is another difference exists between the aforementioned effect of the fatty acyl chain length of monounsaturated monoglycerides on the structure and that of $\omega$-3 PUFA monoglycerides. It appears when comparing the structures of MAG-DPA and MAG-EPA in excess water that their acyl chain lengths have no significant influence as both lipids exhibited $\mathrm{H}_{2}$ phase with almost same lattice parameter. However, we do not exclude that the reported presence of small fraction of free fatty acids (FFAs) in MAG-EPA could affect the lattice parameter of its $\mathrm{H}_{2}$ phase ${ }^{19}$.

To shed light on the effect of the unsaturation degree on the structural features of $\omega-3$ PUFA monoglycerides, MAG-DHA and MAG-DPA were compared with the monounsaturated monoglyceride monoerucin (a monoglyceride of C22:1) that has same fatty acyl chain length. It is interesting that monoerucin also displays $\mathrm{H}_{2}$ phase in excess water with a lattice parameter of about 6.43 and $5.01 \mathrm{~nm}$ at 35 and $90{ }^{\circ} \mathrm{C}$, respectively ${ }^{40}$. For monoerucin, the transition temperatures from dominated crystalline lamellar $\left(\mathrm{L}_{\mathrm{C}}\right)$ phase to $\mathrm{H}_{2}$ and $\mathrm{L}_{2}$ phases in excess water occur at about 33.5 and $129.7^{\circ} \mathrm{C}$, respectively ${ }^{40}$. However, the counterparts MAG-DHA and MAG-DPA with a higher unsaturation degree exhibited as discussed below only $\mathrm{H}_{2}$ phase in excess water at low and ambient temperatures and the transition to $\mathrm{L}_{2}$ phase was detected at temperatures of 54.6 and $75.0{ }^{\circ} \mathrm{C}$, respectively. This significant influence of increasing the unsaturation degree on lowering phase transition temperatures is consistent with previous studies ${ }^{29,35}$. 
Another important remark that can be drawn from the aforementioned comparison between MAGDHA, MAG-DPA and monoerucin is the influence of the unsaturation degree on the lattice parameter of the $\mathrm{H}_{2}$ phase. Increasing the unsaturation degree was associated with a decrease in the corresponding lattice parameter of the $\mathrm{H}_{2}$ phase, which is most likely attributed to narrowing of the hydrophilic nanochannels of the $\mathrm{H}_{2}$ phase due to the generation of more wedged shaped (more disordered) amphiphilic compounds with increasing the number of double bonds on the carbon atom backbones of these monoglycerides. However, it is still important to emphasize that we do not exclude the possible presence of impurities in monoerucin leading to the formation of $\mathrm{H}_{2}$ phase with a greater lattice parameter in excess water than that of MAG-DHA and MAG-DPA.

3.2 Hydration-Triggered Structural Alterations in MAG-DHA Self Assemblies. To investigate the role of hydration on the structural properties of MAG-DHA membrane, three synchrotron SAXS experiments were performed at $37{ }^{\circ} \mathrm{C}$ on binary MAG-DHA/water mixtures containing the following water concentrations: 15, 25 and $40 \mathrm{wt} \%$. The first two samples were clear solutions prepared under limited hydration condition; whereas the third sample was biphasic: fully hydrated MAG-DHA in excess water. At $15 \mathrm{wt} \%$ water, the detected broad peak at $q$ of $\sim 1.93 \mathrm{~nm}^{-1}$ in the presented SAXS pattern (Figure 2B) indicated the formation of inverse micelles $\left(\mathrm{L}_{2}\right.$ phase) with a characteristic distance of about $3.3 \mathrm{~nm}$. Increasing water content to $25 \mathrm{wt} \%$ led to a structural transition to an inverse hexagonal $\left(\mathrm{H}_{2}\right)$ phases with a lattice parameter of $4.66 \mathrm{~nm}$ (Table 1). A further increasing in water content to $40 \mathrm{wt} \%$ did not affect the lattice parameter of the $\mathrm{H}_{2}$ phase (Table 1). This was consistent with previous reports ${ }^{19,29,39-41}$ on the independence of inverse selfassembled nanostructures based on unsaturated monoglycerides, phospholipids, phytantriol, among others, as approaching their maximum water solubilization capacity (the hydration limit). Taken into account that the $\mathrm{H}_{2}$ phase was not affected by increasing water concentration from 25 to 40 $\mathrm{wt} \%$, it was possible to provide an estimate of around $25 \mathrm{wt} \%$ for the maximum water uptake of 
MAG-DHA under full hydration condition at $37^{\circ} \mathrm{C}$. It is interesting that a similar maximum water uptake of about $24 \mathrm{wt} \%$ was reported for the counterpart monoerucin at $33{ }^{\circ} \mathrm{C}^{40}$. This indicates that increasing the unsaturation degree (MAG-DHA vs. monoerucin) has no significant impact on the maximum water uptake of the $\mathrm{H}_{2}$ phase based on highly wedge-shaped amphiphilic compounds as discussed above. However, shortening the fatty acyl chain seems to play an important role in modulating the maximum water uptake of the $\mathrm{H}_{2}$ phase as the counterpart MAG-EPA with a shorter fatty chain length was able to swell larger amount of water (about $40 \mathrm{wt} \%$ at $37^{\circ} \mathrm{C}$ ) ${ }^{19}$.

\subsection{Temperature-Dependence of MAG-DHA and MAG-DPA Self Assemblies in Excess}

Water. A set of SAXS experiments was undertaken to investigate the effect of temperature and examine the reversibility of fully hydrated self-assembled nanostructures of MAG-DHA and MAGDPA by applying a heating-cooling cycle in the temperature range of $5-60{ }^{\circ} \mathrm{C}$ at a ramp rate of 1 ${ }^{\circ} \mathrm{C} / \mathrm{min}$. For both samples, Figure 3 shows selected SAXS patterns taken during the heating stage. Clearly, a similar temperature-triggered $\mathrm{H}_{2}-\mathrm{L}_{2}$ phase transition order was detected for MAG-DHA and MAG-DPA in excess water to that recently reported for MAG-EPA ${ }^{19}$. it was evident from Figure 3A that MAG-DHA in excess water forms stable neat $\mathrm{H}_{2}$ phase in the temperature range of 5- $51.5{ }^{\circ} \mathrm{C}$ and the temperature increase in this range led to a slight decrease in its corresponding lattice parameter from 4.93 to $4.52 \mathrm{~nm}$ with a rate of about $-0.011 \mathrm{~nm} /{ }^{\circ} \mathrm{C}$ (Figure $4 \mathrm{~A}$ ). At $52.6^{\circ} \mathrm{C}$, the three characteristic Bragg peaks of the $\mathrm{H}_{2}$ phase were still detected but an additional board peak at $q \sim 1.61 \mathrm{~nm}^{-1}$ was observed indicating the occurrence of coexisting $\mathrm{L}_{2}$ phase. The structural transition to a neat $\mathrm{L}_{2}$ phase was detected at $54.6{ }^{\circ} \mathrm{C}$ and heating to $60{ }^{\circ} \mathrm{C}$ induced a decrease in its corresponding characteristic distance (Figure 4A) from 3.87 to $3.76 \mathrm{~nm}$ with a rate of about -0.006 $\mathrm{nm} /{ }^{\circ} \mathrm{C}$.

Figure 3B presented selected SAXS patterns taken during the heating stage for MAG-DPA in excess water. As compared to the counterpart MAG-DHA, the main difference as discussed above 
was the significant impact of the unsaturation degree on the $\mathrm{H}_{2}-\mathrm{L}_{2}$ phase transition temperature. Both MAG-DHA and MAG-EPA ${ }^{19}$ has a comparable phase transition temperature of about 54.6 and $56{ }^{\circ} \mathrm{C}$, respectively; whereas the phase transition was detected at about $75{ }^{\circ} \mathrm{C}$ for MAG-DPA. This is consistent with previous reports ${ }^{29,32}$ on lowering phase transitions temperatures with increasing the unsaturation degree. For instance, MO in excess water ${ }^{32}$ has cubic $P n 3 m-\mathrm{H}_{2}$ and $\mathrm{H}_{2}-$ $\mathrm{L}_{2}$ phase transition temperatures of about $95^{\circ} \mathrm{C}$ and above $100^{\circ} \mathrm{C}$, respectively; whereas the counterpart MLO (a monoglyceride of $\mathrm{C} 18: 2$, c6 and c9) ${ }^{29}$ with a greater unsaturation degree (an additional cis double bond at position 6) has a lower temperature for the same transitions of about $54{ }^{\circ} \mathrm{C}$ and $94{ }^{\circ} \mathrm{C}$ respectively. Thus, comparison of the temperature-dependent behavior of MAGDHA with MAG-DPA and monoerucin in excess water indicates that increasing the unsaturation degree is associated with a decrease in the dominance of the $\mathrm{H}_{2}$ phase and the stabilization of the $\mathrm{L}_{2}$ phase at lower temperatures. In addition to the effect of unsaturation degree, it is worth noting that the temperature-dependent behavior of various unsaturated monoglycerides in excess water was discussed in previous reports and found to be sensitive to their molecular structural features: the double bond position ${ }^{31,32}$, the acyl chain length ${ }^{35,36,42}$, the configuration in the acyl chain ${ }^{38,43}$, and the nature of the hydrophilic headgroup ${ }^{44,45}$.

In the temperature range of $5-74{ }^{\circ} \mathrm{C}$, heating of MAG-DPA in excess water induced a decrease in the lattice parameter of the detected neat $\mathrm{H}_{2}$ phase from 5.34 to $4.60 \mathrm{~nm}$ with a rate of about -0.01 $\mathrm{nm} /{ }^{\circ} \mathrm{C}$ (Figure 4B). It should be pointed out that the decrease rate in the lattice parameter of the $\mathrm{H}_{2}$ phase for both MAG-DHA and MAG-DPA are close and similar to that recently reported for MAG$\mathrm{EPA}^{19}$ (about $-0.012 \mathrm{~nm} /{ }^{\circ} \mathrm{C}$ ) and slightly lower than that reported for $\mathrm{MO}\left(-0.016 \mathrm{~nm} /{ }^{\circ} \mathrm{C}\right.$ at the temperature range of about $90-95{ }^{\circ} \mathrm{C}^{43,46}$ ). The detected phase transitions for the investigated $\omega-3$ PUFA monoglycerides in excess water (Figure 3) are attributed to changes in their molecular structural features: a combination of temperature-induced dehydration effect (a decrease in the 
hydration degree of their hydrophilic headgroups) and an increase in the thermal expansivities (enhancement of the effective volume of their fatty acyl chains) $)^{19,29,32,47,48}$.

For gaining insight into the reversibility/irreversibility of the structural transition, the temperaturedependent behavior was also investigated during the cooling stage. Selected SAXS patterns (red colored curves) are presented during the heating stage and compared to those obtained at same temperature (black colored curves) during cooling (Figure 5). For the MAG-DPA sample, a slight hysteresis similar to that reported to $\mathrm{MAG-EPA}{ }^{19}$ was detected and attributed to dynamic structural transition under non-equilibrium conditions. As discussed in a previous review" ${ }^{49}$ on 'highly cooperative' phase transitions, the occurrence of such a slight hysteresis is most likely attributed to the application of experimental time scales shorter than the equilibrium time scales for the transition regions in these lipid systems. In this group of lipid systems characterized with fast phase transitions, reversibility can be achieved when the applied experimental time scales are sufficient for (i) the re-arrangements of the lipid molecules in excess water, and (ii) the re-establishment of the hydrogen bonding networks around the hydrophilic headgroups of the lipids during the heatingcooling cycle ${ }^{19,49}$. For MAG-DHA sample, it is worth noting that the obtained SAXS results in Figures 4 and $\mathbf{5}$ indicate a more significant hysteresis as compared to that of MAG-DPA and MAGEPA $^{19}$. Further SAXS experiments are required to gain insight into the effects of the unsaturation degree and the fatty acyl chain length of $\omega-3$ PUFA monoglycerides on the reversibility/irreversibility of the structural transitions by applying different equilibration times during the cooling-heating cycles.

For MAG-DHA, the lattice parameter of the $\mathrm{H}_{2}$ phase (Figure 4A) during the cooling stage was increased from 4.50 to $4.87 \mathrm{~nm}$ with a rate of $0.008 \mathrm{~nm} /{ }^{\circ} \mathrm{C}$ in the temperature range of $49.2-4.8^{\circ} \mathrm{C}$, and therefore similar to that observed during the heating stage as shown above. An additional intermediate $\mathrm{H}_{2}$ phase with a lattice parameter in the range of 4.45-4.74 nm was also detected on 
decreasing the temperature from 42.3 to $4.8^{\circ} \mathrm{C}$, along with a same increasing rate of about 0.008 $\mathrm{nm} /{ }^{\circ} \mathrm{C}$. It should be noted that a similar occurrence of an intermediate $\mathrm{H}_{2}$ phase was reported during the heating stage of MAG-EPA ${ }^{19}$. Further, the SAXS patterns do not only indicate the occurrence of an intermediate $\mathrm{H}_{2}$ phase as mentioned above, but the detection of a mixture of different weak Bragg peaks with an associated broadening of the characteristic Bragg peaks of the $\mathrm{H}_{2}$ phase (Figure 5A) is most likely attributed to the uptake of water at different rates into the water domains during the cooling stage, giving rise to this more complex pattern. The temperature-dependent behavior of MAG-DPA (Figure 4B) was slightly different as the lattice parameter of the $\mathrm{H}_{2}$ phase during the cooling stage was changed almost with an identical rate (about $0.011 \mathrm{~nm} /{ }^{\circ} \mathrm{C}$ ) to that obtained during heating.

\subsection{Formation, Characterization, and Temperature-Triggered Phase Transitions on MAG-} DHA and MAG-DPA Nano-Self Assemblies. Three MAG-DHA nanodispersions were prepared by using the tri-block copolymer Pluronic ${ }^{\circledR}$ F127 or the anionic food-grade emulsifier citrem as stabilizer and characterized by SAXS (Figure 6A). Pluronic ${ }^{\circledR}$ F127, known also as Poloxamer 407, is the most investigated stabilizer for cubosomes and hexosomes and other related nanodispersions $^{20,26,50}$. Citrem, a FDA-approved food-grade anionic emulsifier of citric acid ester of monoglycerides and diglycerides, was selected in the present study as immune safe stabilizer for non-lamellar liquid crystalline nanoparticles with potential application in the development of injectable hemocompatible nanocarriers as recently reported ${ }^{28,51}$. For F127-stablized nanodispersion, the $\mathrm{H}_{2}$ phase was retained in the nanoparticles' interiors as the corresponding lattice parameter of $4.66 \mathrm{~nm}$ at $37{ }^{\circ} \mathrm{C}$ was identical to that of the bulk (non-dispersed) fully hydrated $\mathrm{H}_{2}$ phase (Table 1). This indicates that F127 is an efficient stabilizer mainly adhering hexosomes without infecting their internal nanostructures. This consistent with previous studies on the use of F127 as stabilizer of hexosomes based on unsaturated monoglycerides ${ }^{19,29,44,52}$. It should be pointed 
out that the enlargement of the hydrophilic nanochannels of the $\mathrm{H}_{2}$ phase could be associated with structural alterations in the internal $\mathrm{H}_{2}$ phase of hexosomes as previously reported for hexosomes based on the binary MLO/diglycerol monooleate (DGMO) mixtures ${ }^{44}$. The broadening of the characteristic Bragg peaks for the internal $\mathrm{H}_{2}$ phase in hexosomes (Figure 6A) as compared to the corresponding $\mathrm{H}_{2}$ phase of the fully hydrated (non-dispersed) MAG-DHA sample was previously observed for F127-stablized cubosomes and hexosomes based on $\mathrm{MLO}^{29}$ and attributed to the presence of vesicles and possible coexistence of other small aggregates. The formation of hexosomes coexisting with vesicles was further confirmed by cryo-TEM (Figure 7A-C). Based on SAXS experiments and cryo-TEM observations, a similar behavior was also observed for the F127stablized MAG-DPA nanodispersion (Figure 6B) and it was consistent with the recent report on formation of hexosomes based on MAG-EPA ${ }^{19}$. The $\mathrm{H}_{2}$ phase with a lattice parameter of $5.01 \mathrm{~nm}$ was retained in excess water at $37{ }^{\circ} \mathrm{C}$ (Table 1) after its fragmentation into hexosomes in the presence of F127. In Figure 7, the cryo-TEM images of both MAG-DHA (Figure 7A-C) and MAG-DPA (Figure 7D, E) hexosomes exhibited the typical morphological features ${ }^{29}$ of surfaces resembling hexagons (marked with a white arrow) or curved striation (marked with an asterisk). The observed vesicular structures including multi-lamellar vesicles were marked with red arrows.

For the two citrem-stabilized nanodispersions prepared at citrem/MAG-DHA weight ratios of 2:3 and 3:2, the $\mathrm{H}_{2}$ phase of MAG-DHA in excess water was affected post-fragmentation into colloidal nanoparticles. At citrem/MAG-DHA weight ratio of 2:3, the incorporation of citrem into the nanoparticles' interiors led to functionalization of the internal $\mathrm{H}_{2}$ phase (enlargement of the hydrophilic nanochannels) with an increase in the lattice parameter from 4.66 to $4.86 \mathrm{~nm}$ (Table 1). Increasing citrem/MAG-DHA weight ratio to $3: 2$ led to a further increase of lattice parameter to about $5.06 \mathrm{~nm}$, but also an additional broad peak appeared at $q \sim 1.5 \mathrm{~nm}^{-1}$ indicating the occurrence of a coexisting less ordered internal $\mathrm{L}_{2}$ phase. These structural alterations on using citrem as 
stabilizer for non-lamellar liquid crystalline nanoparticles based on commercial MO (or its combination with an oil) and MAG-EPA was discussed in previous studies ${ }^{19,28,51}$, and attributed to its penetration to the hydrophobic domains of the nanoparticles' self-assembled interiors ${ }^{28,51,53,54}$.

It should be pointed out that citrem was tested as a potential stabilizer for MAG-DPA nanodispersions at the aforementioned citrem/monoglyceride ratios. However, it was not efficient and considerable amount of MAG-DPA was not fully dispersed in excess water. This is most likely attributed to a significant decrease in the fraction of citrem molecules that adhere to the outer surfaces of the dispersed nanoparticles. In such ionic dispersions, the colloidal stabilization occurs via electrostatic effects and requires sufficient citrem surface coverage on the dispersed nanoparticles in excess water. In addition to SAXS and cryo-TEM, NTA was used to gain insight into the nanoparticle size distribution of the investigated nanodispersions. For both MAG-DHA and MAG-DPA nanodispersions stabilized using F127, the mean (diameters) and mode sizes were in the range of 88.6-95.6 and 81.4-84.0 $\mathrm{nm}$, respectively, and comparable with those recently reported for MAG-EPA at same stabilizer concentration (Table 1). Citrem is less efficient than the steric stabilizer F127 and therefore larger mean nanoparticle sizes (diameters) and modes were obtained (Table 1). These findings are consistent with previous studies on the electrostatic stabilization of nanoparticles based MAG-EPA ${ }^{19}$ and the binary MO/medium chain triglycerides (MCT) mixture ${ }^{28}$ by using citrem. The zeta potential of citrem-stabilized nanodispersions were in the range of -45.7 to $-46.17 \mathrm{mV}$. With increasing citrem content in MAG-DHA nanodispersions, a marginally decreased negative zeta potential obtained, which is also in good agreement with previous studies using the anionic stabilizer citrem for stabilization of non-lamellar liquid crystalline nanoparticles $^{19,28}$.

The temperature-dependent behavior of MAG-DHA and MAG-DPA nanodispersions stabilized by F127 was also investigated. Figure 8A shows selected SAXS patterns of MAG-DHA 
nanodispersion during the heating-cooling cycle between 5 to $60{ }^{\circ} \mathrm{C}$ with a temperature ramp of $1{ }^{\circ} \mathrm{C}$ /min. It is evident that the internal structure of MAG-DHA nanoparticles underwent a structural transition from $\mathrm{H}_{2}$ phase (hexosomes) to an internal $\mathrm{L}_{2}$ phase (emulsified $\mathrm{L}_{2}$ phase nanoparticles, ELPs) during the heating stage, in analogy to that observed for the non-dispersed fully hydrated MAG-DHA sample. In the temperature range of $5-46.4{ }^{\circ} \mathrm{C}$, the lattice parameter of the internal $\mathrm{H}_{2}$ phase dropped from 5.06 to $4.60 \mathrm{~nm}$ with a rate of $-0.011 \mathrm{~nm} /{ }^{\circ} \mathrm{C}$ that was identical to that of the corresponding bulk (non-dispersed) fully hydrated MAG-DHA phase and similar to that reported for MAG-EPA hexosomes ${ }^{19}$. A transition to an internal neat $\mathrm{L}_{2}$ phase was found in the temperature range of 49.4 to $60{ }^{\circ} \mathrm{C}$ and its corresponding characteristic distance was decreased with a rate of about $-0.003 \mathrm{~nm} /{ }^{\circ} \mathrm{C}$. In the cooling stage, the lattice parameter of the internal $\mathrm{H}_{2}$ phase was increased with decreasing the temperature from 35.3 to $5.9{ }^{\circ} \mathrm{C}$ and the rate was almost identical to that obtained during heating (Figure 8B). It is worth noting that the increased rate in the lattice parameter of the internal $\mathrm{H}_{2}$ phase $\left(0.011 \mathrm{~nm} /{ }^{\circ} \mathrm{C}\right)$ differed from that of its corresponding fully hydrated bulk (non-dispersed) sample $\left(0.008 \mathrm{~nm} /{ }^{\circ} \mathrm{C}\right)$. This could be attributed to the presence of an intermediate $\mathrm{H}_{2}$ phase during the heating stage of the fully hydrated bulk sample that was not detected in MAG-DHA nanodispersion.

A similar temperature-dependent behavior was also found for F127-stabilized MAG-DPA nanodispersion (Figure 9A, B). As illustrated in Figure 9B, the lattice parameter reduction for the $\mathrm{H}_{2}$ phase was with same rate of about $0.01 \mathrm{~nm} /{ }^{\circ} \mathrm{C}$ in both heating and cooling stages and was identical to that found for the fully hydrated bulk MAG-DPA sample.

It is worth mentioning that the $\mathrm{H}_{2}-\mathrm{L}_{2}$ phase transition temperature for the non-dispersed fully hydrated MAG-DHA sample $\left(54.6^{\circ} \mathrm{C}\right)$ was slightly higher than that for the dispersed sample $(46.4$ ${ }^{\circ} \mathrm{C}$ ). This phenomenon is consistent with previous reported studies on inverse non-lamellar liquid crystalline phases based on unsaturated monoglycerides in the bulk and dispersed states ${ }^{19,29,39,55}$. For 
instance, it was reported that the fragmentation of $\mathrm{MLO}^{29}$ and MAG-EPA ${ }^{19}$ liquid crystalline phases into nanoparticles was associated with a decrease in the $\mathrm{H}_{2}-\mathrm{L}_{2}$ phase transition temperature from 94 to $87^{\circ} \mathrm{C}$, and from 56 to $53.6^{\circ} \mathrm{C}$, respectively. As discussed in our recent report, this is most likely attributed to faster molecular motions of the lipid and water molecules in the dispersed state as compared to those of the corresponding non-dispersed phases. In addition, the presence of F127 in the nanodispersions could facilitate the phase transition at slightly lower temperature ${ }^{19}$.

The aforementioned lack of influence of $\mathrm{F} 127$ on the internal $\mathrm{H}_{2}$ and $\mathrm{L}_{2}$ phases of the nanoparticles was evident from the direct comparison between the structural features of the fully hydrated (nondispersed) samples and the corresponding F127-stabilized nanodispersions of MAG-DHA and MAG-DPA during heating and cooling stages (Figure 10).

\section{CONCLUSIONS}

The experimental findings reveal the important roles of the unsaturation degree and the fatty acyl chain length of $\omega-3$ PUFA monoglycerides on modulating the structural features of their selfassemblies in excess water, and controlling the maximum water solubilization capacity and the structural phase transition temperatures. As compared to monounsaturated monoglycerides with shorter fatty acyl chain lengths such as monoolein, $\omega-3$ PUFA monoglycerides are more wedgedshaped amphiphiles and have, therefore, a propensity to form $\mathrm{H}_{2}$ phase in excess water at room temperature. We found that increasing their unsaturation degree is associated with a significant decrease in the corresponding lattice parameter of the $\mathrm{H}_{2}$ phase and leads to a significant reduction in the $\mathrm{H}_{2}-\mathrm{L}_{2}$ phase transition temperature.

Similar to MAG-EPA, both new amphiphilic $\omega$-3 PUFA monoglycerides, namely MAG-DHA and MAG-DPA, tend to form dominant $\mathrm{H}_{2}$ phase in excess water at low and ambient temperatures and 
this phase can be fragmented in the presence of F127 or citrem to form hexosomes. In combination with NTA and cryo-TEM, synchrotron SAXS was used for verifying the structural features, and the size and morphological features of the produced nanoparticles. In addition, the temperature dependence as well as the influence of the used stabilizers on the structural properties of these nanodispersions are discussed. Taking into account the sensitivity of PUFA compounds to oxidation, the stability against oxidation of $\omega$-3 PUFA monoglycerides, in both dispersed and nondispersed states of the self-assemblies, should be evaluated in future investigations. For citremstabilized nanodispersions, it is well known that citrem has an antioxidant effect, and therefore it is interesting to investigate a possible role for citrem in enhancing the stability against oxidation of hexosomes based on these $\omega-3$ PUFA monoglycerides.

In both dispersed and non-dispersed states, the beneficial health effects of this new family of $\omega-3$ PUFA monoglycerides as well as the unique structural properties of their self-assemblies in excess water provide an efficient approach for the design of new platforms for delivering $\omega-3$ PUFAs alone or in combination with nutraceuticals or therapeutic agents.

\section{CONFLICT OF INTERESTS}

The authors declare no competing financial interest.

\section{ACKNOWLEDGMENTS}

Financial support to AY by the Danish Council for Independent Research | Technology and Production Sciences (references 1335-00150b and DFF- 7017-00065) is gratefully acknowledged. AY further acknowledges financial support from the Danish Natural Sciences Research Council (DanScatt) for SAXS experiments. 
Table 1: Structure parameters (the lattice parameter, $a$, for the $\mathrm{H}_{2}$ phase and the characteristic distance, $d$, for the $\mathrm{L}_{2}$-phase) and NTA size characteristics for the nanodispersions stabilized by F127 or citrem. The structure parameters of the detected self assemblies were derived from SAXS patterns presented in Figure 6.

\begin{tabular}{|c|c|c|c|c|c|c|c|c|c|}
\hline \multirow{2}{*}{ Lipid } & \multirow{2}{*}{$\begin{array}{l}\text { Investigated } \\
\text { system }\end{array}$} & \multirow{2}{*}{$\begin{array}{l}\text { Water } \\
\text { content } \\
(\mathbf{w t} \%)\end{array}$} & \multirow{2}{*}{$\begin{array}{l}\text { Stabilizer } \\
\text { concentrat } \\
\text { ion }(w t \%) \\
\end{array}$} & \multirow{2}{*}{ Phase } & \multirow{2}{*}{$\begin{array}{c}a \\
(\mathbf{n m})\end{array}$} & \multirow{2}{*}{$\begin{array}{c}d \\
(\mathrm{~nm})\end{array}$} & \multicolumn{3}{|c|}{ Size (nm) with Standard Deviation } \\
\hline & & & & & & & Mean & Median & Mode \\
\hline \multirow{6}{*}{$\begin{array}{c}\text { MAG- } \\
\text { DHA }\end{array}$} & \multirow{3}{*}{$\begin{array}{c}\text { Bulk (non- } \\
\text { dispersed): MAG- } \\
\text { DHA/water system }\end{array}$} & 15 & - & $\mathrm{L}_{2}$ & - & 3.25 & & & \\
\hline & & 25 & - & $\mathrm{H}_{2}$ & 4.66 & - & & & \\
\hline & & $40^{\mathrm{a}}$ & - & $\mathrm{H}_{2}$ & 4.66 & - & & & \\
\hline & $\begin{array}{c}\text { F127-stablized } \\
\text { dispersion (sample } \\
\text { DF127) }\end{array}$ & 94 & 1 & $\mathrm{H}_{2}$ & 4.66 & - & $92.1 \pm 3.5$ & $81.5 \pm 2.5$ & $82.7 \pm 1.3$ \\
\hline & $\begin{array}{c}\text { Citrem-stabilized } \\
\text { dispersion (sample } \\
\text { CM2:3) } \\
\end{array}$ & 95 & 2 & $\mathrm{H}_{2}$ & 4.86 & - & $130.6 \pm 0.6$ & $111.7 \pm 0.1$ & $101.1 \pm 2.8$ \\
\hline & $\begin{array}{c}\text { Citrem-stabilized } \\
\text { dispersion (sample } \\
\text { CM3:2) }\end{array}$ & 95 & 3 & $\begin{array}{r}\mathrm{H}_{2} \\
\mathrm{~L}_{2}\end{array}$ & $\begin{array}{c}5.06 \\
-\end{array}$ & 4.4 & $124.7 \pm 0.8$ & $111.9 \pm 1.4$ & $115.6 \pm 5.0$ \\
\hline \multirow{2}{*}{$\begin{array}{c}\text { MAG- } \\
\text { DPA }\end{array}$} & $\begin{array}{c}\text { Bulk (non- } \\
\text { dispersed): MAG- } \\
\text { DPA/water system }\end{array}$ & $90^{\mathrm{a}}$ & - & $\mathrm{H}_{2}$ & 5.00 & - & & & \\
\hline & $\begin{array}{c}\text { F127-stablized } \\
\text { dispersion (sample } \\
\text { DF127) }\end{array}$ & 94 & 1 & $\mathrm{H}_{2}$ & 5.01 & - & $106.5 \pm 1.8$ & $97.5 \pm 1.6$ & $97.9 \pm 5.1$ \\
\hline \multirow{4}{*}{$\begin{array}{l}\text { MAG- } \\
\text { EPA }^{\mathrm{d}}\end{array}$} & $\begin{array}{c}\text { Bulk (non- } \\
\text { dispersed): MAG- } \\
\text { EPA/water system }\end{array}$ & $90^{\mathrm{a}}$ & - & $\mathrm{H}_{2}$ & 4.87 & - & & & \\
\hline & $\begin{array}{c}\text { F127-stablized } \\
\text { dispersion (sample } \\
\text { DF127) }\end{array}$ & 94 & 1 & $\mathrm{H}_{2}$ & 4.87 & - & $82.0 \pm 30.3$ & $72.7 \pm 30.3$ & $66.3 \pm 30.3$ \\
\hline & $\begin{array}{c}\text { Citrem-stabilized } \\
\text { dispersion (sample } \\
\text { CM2:3) }\end{array}$ & 95 & 2 & $\mathrm{H}_{2}$ & 4.91 & - & $128.7 \pm 51.3$ & $115.6 \pm 51.3$ & $97.8 \pm 51.3$ \\
\hline & $\begin{array}{c}\text { Citrem-stabilized } \\
\text { dispersion (sample } \\
\text { CM3:2) }\end{array}$ & 95 & 3 & $\begin{array}{l}\mathrm{H}_{2} \\
\mathrm{~L}_{2}\end{array}$ & $\begin{array}{c}5.10 \\
-\end{array}$ & 4.4 & $114.3 \pm 40.0$ & $104.1 \pm 40.0$ & $90.7 \pm 40.0$ \\
\hline
\end{tabular}

${ }^{\mathrm{a}}$ Fully hydrated MAG-DHA and MAG-DPA samples.

${ }^{\mathrm{b}}$ The nanodispersion was prepared at citrem $/ \omega-3$ PUFA monoglyceride weight ratio of $2: 3$.

${ }^{\mathrm{c}}$ The nanodispersion was prepared at citrem/ $/ \omega-3$ PUFA monoglyceride weight ratio of $3: 2$.

${ }^{\mathrm{d}}$ The results for the MAG-EPA fully hydrated sample and the three corresponding nanodispersions were taken from Yaghmur et al. ${ }^{19}$. 


\section{REFERENCES}

1 W. Wang, J. Zhu, F. Lyu, D. Panigrahy, K. W. Ferrara, B. Hammock and G. Zhang, $\omega-3$ Polyunsaturated fatty acids-derived lipid metabolites on angiogenesis, inflammation and cancer, Prostaglandins Other Lipid Mediat., 2014, 113-115, 13-20.

2 R. Khaddaj-Mallat, C. Morin and E. Rousseau, Novel n-3 PUFA monoacylglycerides of pharmacological and medicinal interest: Anti-inflammatory and anti-proliferative effects, Eur. J. Pharmacol., 2016, 792, 70-77.

3 C. Morin, E. Rodríguez, P. U. Blier and S. Fortin, Potential application of eicosapentaenoic acid monoacylglyceride in the management of colorectal cancer, Mar. Drugs, 2017, 15, 283.

4 C. Morin, R. Hiram, E. Rousseau, P. U. Blier and S. Fortin, Docosapentaenoic acid monoacylglyceride reduces inflammation and vascular remodeling in experimental pulmonary hypertension, AJP Hear. Circ. Physiol., 2014, 307, H574-H586.

5 C. Morin, E. Rousseau, P. U. Blier and S. Fortin, Effect of docosahexaenoic acid monoacylglyceride on systemic hypertension and cardiovascular dysfunction, Am. J. Physiol. - Hear. Circ. Physiol., 2015, 309, H93-H102.

6 C. Morin, E. Rousseau and S. Fortin, Anti-proliferative effects of a new docosapentaenoic acid monoacylglyceride in colorectal carcinoma cells, Prostaglandins, Leukot. Essent. Fat. Acids, 2013, 89, 203-213.

7 C. N. Serhan, Pro-resolving lipid mediators are leads for resolution physiology, Nature, 2014, 510, 92-101.

$8 \quad$ S. Fortin, US Pat., 8119690 B2, 2012.

9 S. Fortin, US Pat., 8222295 B2, 2012.

10 C. Morin, P. U. Blier and S. Fortin, MAG-EPA reduces severity of DSS-induced colitis in rats, Am. J. Physiol. Liver Physiol., 2016, 310, G808-G821.

11 C. Morin and S. Fortin, Docosahexaenoic acid monoglyceride increases carboplatin activity in lung cancer models by targeting EGFR, Anticancer Res., 2017, 37, 6015-6023.

12 P. M. Kris-Etherton, W. S. Harris and L. J. Appel, Fish consumption, fish oil, omega-3 fatty acids, and cardiovascular disease, Circulation, 2002, 106, 2747-2757.

13 H. K. Maehre, I.-J. Jensen, E. O. Elvevoll and K.-E. Eilertsen, $\omega-3$ Fatty acids and cardiovascular diseases: Effects, mechanisms and dietary relevance, Int. J. Mol. Sci., 2015, 16, 22636-22661.

14 C. Morin, A. M. Cantin, E. Rousseau, M. Sirois, C. Sirois, E. Rizcallah and S. Fortin, Proresolving action of docosahexaenoic acid monoglyceride in lung inflammatory models related to cystic fibrosis, Am. J. Respir. Cell Mol. Biol., 2015, 53, 574-583.

15 C. Morin, S. Fortin, A. M. Cantin and E. Rousseau, MAG-EPA resolves lung inflammation in an allergic model of asthma, Clin. Exp. Allergy, 2013, 43, 1071-1082. 
16 F, Destaillats, C. C. Hernandez, F. Dionisi, I. M. Elmelegy, M. Oliveira and J. C. Moulin, EU Pat., 3135283A1, 2017.

17 N. Tran, X. Mulet, A. Hawley, C. Fong, J. Zhai, T. C. Le, J. Ratcliffe and C. J. Drummond, Manipulating the ordered nanostructure of self-assembled monoolein and phytantriol nanoparticles with unsaturated fatty acids, Langmuir, 2018, 34, 2764-2773.

18 A. Angelova, M. Drechsler, V. M. Garamus and B. Angelov, Liquid crystalline nanostructures as PEGylated reservoirs of omega 3 polyunsaturated fatty acids: Structural insights toward delivery formulations against neurodegenerative disorders, ACS omega, $2018,3,3235-3247$.

19 A. Yaghmur, S. Al-Hosayni, H. Amenitsch and S. Salentinig, Structural investigation of bulk and dispersed inverse lyotropic hexagonal liquid crystalline phases of eicosapentaenoic acid monoglyceride, Langmuir, 2017, 33, 14045-14057.

20 A. Angelova, B. Angelov, V. M. Garamus, P. Couvreur and S. Lesieur, Small-angle X-ray scattering investigations of biomolecular confinement, loading, and release from liquidcrystalline nanochannel assemblies, J. Phys. Chem. Lett., 2012, 3, 445-457.

21 K. Khaliqi, A. Ghazal, I. D. M. Azmi, H. Amenitsch, K. Mortensen, S. Salentinig and A. Yaghmur, Direct monitoring of lipid transfer on exposure of citrem nanoparticles to an ethanol solution containing soybean phospholipids by combining synchrotron SAXS with microfluidics, Analyst, 2017, 142, 3118-3126.

22 C. Nilsson, B. Barrios-Lopez, A. Kallinen, P. Laurinmäki, S. J. Butcher, M. Raki, J. Weisell, K. Bergström, S. W. Larsen, J. Østergaard, C. Larsen, A. Urtti, A. J. Airaksinen and A. Yaghmur, SPECT/CT imaging of radiolabeled cubosomes and hexosomes for potential theranostic applications, Biomaterials, 2013, 34, 8491-8503.

23 A. Angelova, V. M. Garamus, B. Angelov, Z. Tian, Y. Li and A. Zou, Advances in structural design of lipid-based nanoparticle carriers for delivery of macromolecular drugs, phytochemicals and anti-tumor agents, Adv. Colloid Interface Sci., 2017, 249, 331-345.

24 V. Meli, C. Caltagirone, A. M. Falchi, S. T. Hyde, V. Lippolis, M. Monduzzi, M. ObiolsRabasa, A. Rosa, J. Schmidt, Y. Talmon and S. Murgia, Docetaxel-loaded fluorescent liquidcrystalline nanoparticles for cancer theranostics, Langmuir, 2015, 31, 9566-9575.

25 I. D. M. Azmi, J. Østergaard, S. Stürup, B. Gammelgaard, A. Urtti, S. M. Moghimi and A. Yaghmur, Cisplatin encapsulation generates morphologically different multicompartments in the internal nanostructures of nonlamellar liquid-crystalline self-assemblies, Langmuir, 2018, 34, 6570-6581.

26 I. D. Azmi, S. M. Moghimi and A. Yaghmur, Cubosomes and hexosomes as versatile platforms for drug delivery, Ther. Deliv., 2015, 6, 1347-1364.

27 M. Gontsarik, M. T. Buhmann, A. Yaghmur, Q. Ren, K. Maniura-Weber and S. Salentinig, Antimicrobial peptide-driven colloidal transformations in liquid-crystalline nanocarriers, $J$. Phys. Chem. Lett., 2016, 7, 3482-3486.

28 I. D. M. Azmi, P. P. Wibroe, L. P. Wu, A. I. Kazem, H. Amenitsch, S. M. Moghimi and A. 
Yaghmur, A structurally diverse library of safe-by-design citrem-phospholipid lamellar and non-lamellar liquid crystalline nano-assemblies, J. Control. Release, 2016, 239, 1-9.

29 L. de Campo, A. Yaghmur, L. Sagalowicz, M. E. Leser, H. Watzke and O. Glatter, Reversible phase transitions in emulsified nanostructured lipid systems, Langmuir, 2004, 20, $5254-5261$.

30 J. Briggs, The phase behavior of hydrated monoglycerides and the design of an X-ray compatible scanning calorimeter, PhD dissertation, The Ohio State University, 1994.

31 H. Qiu and M. Caffrey, Lyotropic and thermotropic phase behavior of hydrated monoacylglycerols: structure characterization of monovaccenin, J. Phys. Chem. B, 1998, 102, 4819-4829.

32 H. Qiu and M. Caffrey, The phase diagram of the monoolein/water system: Metastability and equilibrium aspects, Biomaterials, 2000, 21, 223-234.

33 J. N. Israelachvili, Intermolecular and surface forces, Academic Press, Santa Barbara, 2011.

34 L. V. Misquitta, Y. Misquitta, V. Cherezov, O. Slattery, J. M. Mohan, D. Hart, M. Zhalnina, W. A. Cramer and M. Caffrey, Membrane protein crystallization in lipidic mesophases with tailored bilayers, Structure, 2004, 12, 2113-2124.

35 E. S. Lutton, Phase behavior of aqueous systems of monoglycerides, J. Am. Oil Chem. Soc., $1965,42,1068-1070$.

36 C. Fong, T. Le and C. J. Drummond, Lyotropic liquid crystal engineering-ordered nanostructured small molecule amphiphile self-assembly materials by design, Chem. Soc. Rev., 2012, 41, 1297-1322.

37 T. Kaasgaard and C. J. Drummond, Ordered 2-D and 3-D nanostructured amphiphile selfassembly materials stable in excess solvent, Phys. Chem. Chem. Phys., 2006, 8, 4957.

38 A. Yaghmur, B. Sartori and M. Rappolt, Self-assembled nanostructures of fully hydrated monoelaidin-elaidic acid and monoelaidin-oleic acid systems, Langmuir, 2012, 28, 10105 10119.

39 A. Yaghmur and O. Glatter, Characterization and potential applications of nanostructured aqueous dispersions, Adv. Colloid Interface Sci., 2009, 147-148, 333-342.

40 H. Qiu and M. Caffrey, Phase behavior of the monoerucin/water system, Chem. Phys. Lipids, 1999, 100, 55-79.

41 A. Yaghmur, P. Laggner, M. Almgren and M. Rappolt, Self-assembly in monoelaidin aqueous dispersions: Direct vesicles to cubosomes transition, PLOS ONE, 2008, 3, e3747.

42 J. Briggs and M. Caffrey, The temperature-composition phase diagram and mesophase structure characterization of monopentadecenoin in water, Biophys. J., 1994, 67, 1594-1602.

43 C. Czeslik, R. Winter, G. Rapp and K. Bartels, Temperature- and pressure-dependent phase behavior of monoacylglycerides monoolein and monoelaidin, Biophys. J., 1995, 68, $1423-$ 1429. 
44 A. Yaghmur, L. de Campo, L. Sagalowicz, M. Leser and O. Glatter, Control of the internal structure of MLO-based isasomes by the addition of diglycerol monooleate and soybean phosphatidylcholine, Langmuir, 2006, 22, 9919-9927.

45 M. Johnsson, Y. Lam, J. Barauskas and F. Tiberg, Aqueous phase behavior and dispersed nanoparticles of diglycerol monooleate/glycerol dioleate mixtures, 2005, 21, 5159-5165.

46 A. Yaghmur, P. Laggner, S. Zhang and M. Rappolt, Tuning curvature and stability of monoolein bilayers by designer lipid-like peptide surfactants, PLoS ONE, 2007, 2, e479.

47 A. Yaghmur, L. de Campo, S. Salentinig, L. Sagalowicz, M. E. Leser and O. Glatter, Oilloaded monolinolein-based particles with confined inverse discontinuous cubic structure (Fd3m), Langmuir, 2005, 22, 517-521.

48 A. Yaghmur, L. de Campo, L. Sagalowicz, M. E. Leser and O. Glatter, Emulsified microemulsions and oil-containing liquid crystalline phases, Langmuir 2004, 21, 569-577.

49 B. Tenchov, On the reversibility of the phase transitions in lipid-water systems, Chem. Phys. Lipids, 1991, 57, 165-177.

50 A. J. Tilley, C. J. Drummond and B. J. Boyd, Disposition and association of the steric stabilizer Pluronic ${ }^{\circledR}$ F127 in lyotropic liquid crystalline nanostructured particle dispersions, J. Colloid Interface Sci., 2013, 392, 288-296.

51 P. P. Wibroe, I. D. M. Azmi, C. Nilsson, A. Yaghmur and S. M. Moghimi, Citrem modulates internal nanostructure of glyceryl monooleate dispersions and bypasses complement activation: Towards development of safe tunable intravenous lipid nanocarriers, Nanomedicine: Nanotechnol. Biol. Med., 2015, 11, 1909-1914.

52 M. Nakano, T. Teshigawara, A. Sugita, W. Leesajakul, A. Taniguchi, T. Kamo, H. Matsuoka and T. Handa, Dispersions of liquid crystalline phases of the monoolein/oleic acid/Pluronic F127 system, Langmuir, 2002, 18, 9283-9288.

53 S. F. Hedegaard, C. Nilsson, P. Laurinmäki, S. Butcher, A. Urtti and A. Yaghmur, Nanostructured aqueous dispersions of citrem interacting with lipids and PEGylated lipids, RSC Adv., 2013, 3, 24576.

54 C. Nilsson, K. Edwards, J. Eriksson, S. W. Larsen, J. Østergaard, C. Larsen, A. Urtti and A. Yaghmur, Characterization of oil-free and oil-loaded liquid-crystalline particles stabilized by negatively charged stabilizer Citrem, Langmuir, 2012, 28, 11755-11766.

55 Y.-D. Dong, I. Larson, T. Hanley and B. J. Boyd, Bulk and dispersed aqueous phase behavior of phytantriol: Effect of vitamin E acetate and F127 polymer on liquid crystal nanostructure, Langmuir, 2006, 22, 9512-9518. 


\section{FIGURE CAPTIONS}

Figure 1. The molecular structures of monoolein (MO; 18:1 n-9), mono-10(Z)-heptadecenoin (MHO; a monoglyceride of C17:1), and $\omega$-3 PUFAs monoglycerides: eicosapentaenoic acid (MAGEPA; 20:5 n-3), docosapentaenoic acid (MAG-DPA; 22:5 n-3), and docosahexaenoic acid (MAGDHA; 22:6 n-3) monoglycerides.

Figure 2. (A) SAXS patterns at $25^{\circ} \mathrm{C}$ for the fully hydrated bulk (non-dispersed) non-lamellar liquid crystalline phases based on the following lipids: MHO, MO, MAG-EPA, MAG-DHA, and MAG-DPA. It should be noted that the SAXS pattern of the fully hydrated MAG-EPA samples was taken from Yaghmur et al. ${ }^{19}$. (B) The SAXS patterns of hydrated bulk (non-dispersed) MAG-DHA samples at $37^{\circ} \mathrm{C}$ and three different water concentrations: 15,25 , and $40 \mathrm{wt} \%$. The intensities in both panels have been shifted by a constant arbitrary factor for better visibility.

Figure 3. In the heating direction: selected SAXS patterns at different temperatures for the fully hydrated MAG-DHA (A) and MAG-DPA (B) systems. The patterns were plotted every $5{ }^{\circ} \mathrm{C}$ in the applied scan rate of $1{ }^{\circ} \mathrm{C} / \mathrm{min}$ and temperature ranges of $5-60{ }^{\circ} \mathrm{C}$ and $5-75^{\circ} \mathrm{C}$, respectively.

Figure 4. Variation in the characteristic distance and the lattice parameter as a function of temperature for the $\mathrm{L}_{2}$ and $\mathrm{H}_{2}$ phases, respectively. The data are presented for the fully hydrated MAG-DHA (A) and MAG-DPA (B) systems during the applied heating-cooling cycle. In the heating stage: the $\mathrm{L}_{2}$ and $\mathrm{H}_{2}$ phases are represented by full black circles and full olive colored squares, respectively; whereas they are represented by open red circles, and open olive colored squares during the cooling stage. It should be noted that the detected intermediate $\mathrm{H}_{2}$ phase for MAG-DHA sample under full hydration conditions is represented by inverted full triangles (A).

Figure 5. Selected SAXS patterns for the temperature-dependent behavior of the fully hydrated MAG-DHA (A) and MAG-DPA (B) samples during the heating-cooling cycle in the temperature 
range of $5-60{ }^{\circ} \mathrm{C}$ and a scan rate of $1{ }^{\circ} \mathrm{C} / \mathrm{min}$. In the heating direction, the selected patterns at different temperatures (red lines) are compared with those taken at same temperatures during cooling the samples (black lines).

Figure 6. Comparison of the SAXS patterns of the fully hydrated bulk (non-dispersed) $\mathrm{H}_{2}$ phases of MAG-DHA (A) and MAG-DPA (B) samples with those of their corresponding nanodispersions at $37{ }^{\circ} \mathrm{C}$. For MAG-DHA, the SAXS patterns were taken from F127- and citrem-stabilized nanodispersions (A); whereas MAG-DPA nanodispersion (B) was stabilized by using F127. The two citrem-stabilized nanodispersions presented in (A) were prepared at citrem/MAG-DHA (CM) weight ratios of 2:3 and 3:2. The intensities in (A) and (B) have been shifted by a constant arbitrary factor for better visibility.

Figure 7. Cryo-TEM images of F127-stabilized hexosomes based on MAG-DHA (A-C) and MAGDPA (D and E). Scale bars: $100 \mathrm{~nm}$. In C and E, hexosomes coexisted with vesicular structures including multi-lamellar vesicles (marked with red arrow) were observed. For hexosomes, the detected nanoparticles exhibited either surfaces resembling hexagons (marked with a white arrow in $\mathrm{B}$ and $\mathrm{C}$ ) or curved striations (marked with asterisk in B and $\mathrm{E}$ ).

Figure 8. (A) Representative SAXS patterns for the temperature-dependent behavior of the F127stabilized MAG-DHA nanodispersion during the heating-cooling cycle. In the heating direction, the selected patterns at different temperatures (red lines) are compared with those taken at same temperatures during cooling the samples (black lines). (B) Variation in the characteristic distance and the lattice parameter of the $\mathrm{L}_{2}$ and $\mathrm{H}_{2}$ phases, respectively, as a function of temperature. The data are extracted from the performed SAXS experiments during the heating-cooling circle. During the heating step, the $\mathrm{L}_{2}$ and $\mathrm{H}_{2}$ phases are represented by full black circles and full olive colored 
squares, respectively; whereas they are represented by open red circles and open olive colored squares, respectively, during the cooling step.

Figure 9. (A) Representative SAXS patterns for the temperature-dependent behavior of the F127stabilized MAG-DPA nanodispersion during the heating-cooling cycle. In the heating direction, the selected patterns at different temperatures (red lines) are compared with those taken at same temperatures during cooling the samples (black lines). (B) Variation in the lattice parameter of the $\mathrm{H}_{2}$ phase as a function of temperature. The data are extracted from the performed SAXS experiments during the heating-cooling circle. In the heating and cooling stages, the $\mathrm{H}_{2}$ phase is represented by full and open olive colored squares, respectively.

Figure 10. Variation in the characteristic distance and the lattice parameter of the internal $\mathrm{L}_{2}$ and $\mathrm{H}_{2}$ phases, respectively, as a function of temperature for F127-stabilized dispersions based on MAGDHA (A and B) and MAG-DPA (C and D) as compared to their corresponding fully hydrated bulk (non-dispersed) samples. For MAG-DHA (A and B): the change in the lattice parameter of the $\mathrm{H}_{2}$ phase, and the characteristic distance, $d$, of the $\mathrm{L}_{2}$ phase in the temperature range of $5-60{ }^{\circ} \mathrm{C}$ is given during heating (A) and cooling (B) stages. In C and D, the variation in the lattice parameter of the $\mathrm{H}_{2}$ phase of the dispersed and non-dispersed samples was given in the temperature ranges of 573 and 5-60 ${ }^{\circ} \mathrm{C}$ during the heating and cooling stages, respectively. 


\section{Figure 1}

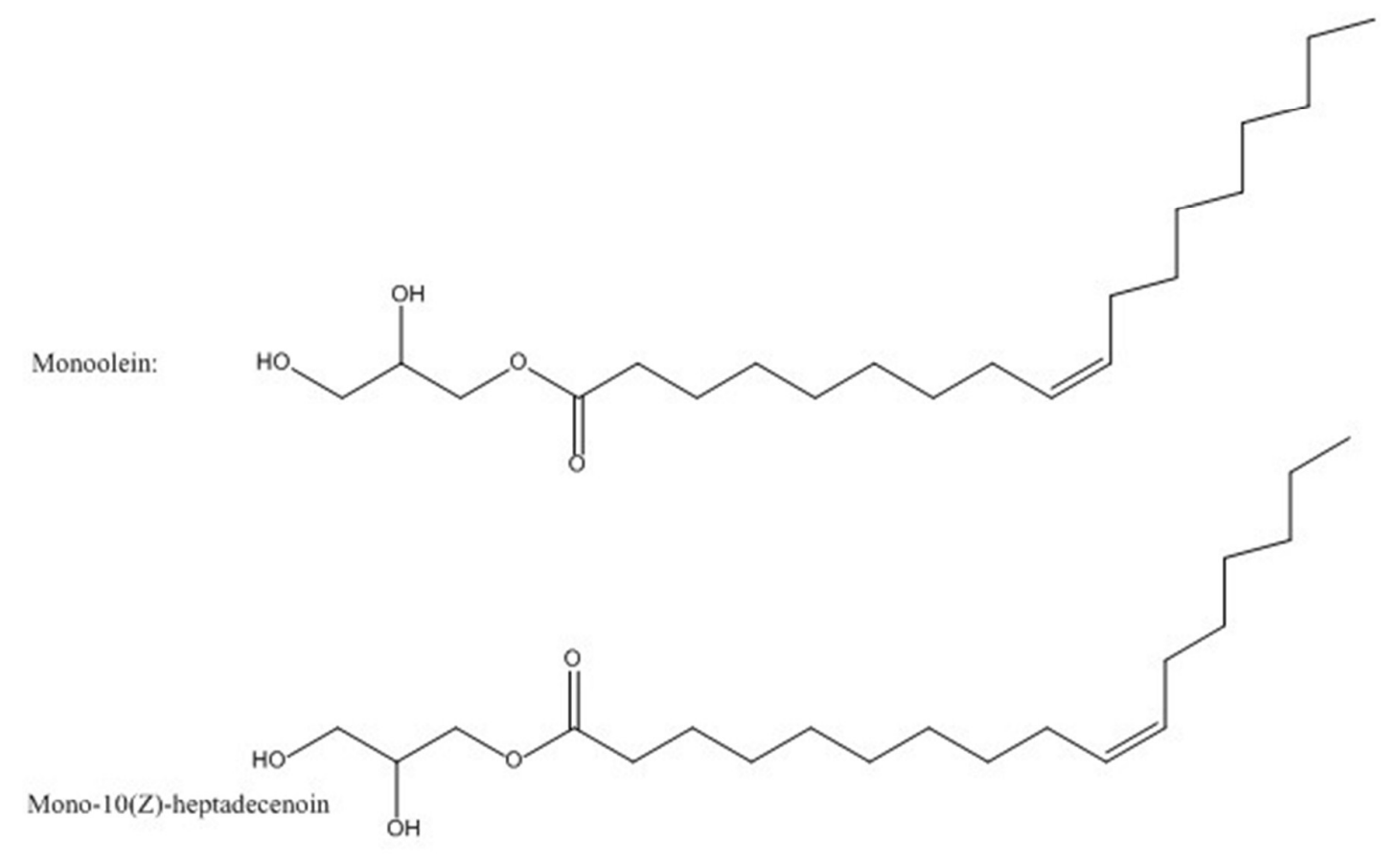

MAG-DHA<smiles>CCCCC/C=C\C/C=C\C/C=C\C/C=C\C/C=C\CCC(=O)OCC(O)CO</smiles>

MAG-EPA<smiles>CC/C=C\C/C=C\C/C=C\C/C=C\C/C=C\CCCC(=O)OCC(O)CO</smiles>

MAG-DPA<smiles>CC/C=C\C/C=C\C/C=C\C/C=C\C/C=C\CCCCCC(=O)OCC(O)CO</smiles> 


\section{Figure 2}

\section{A}
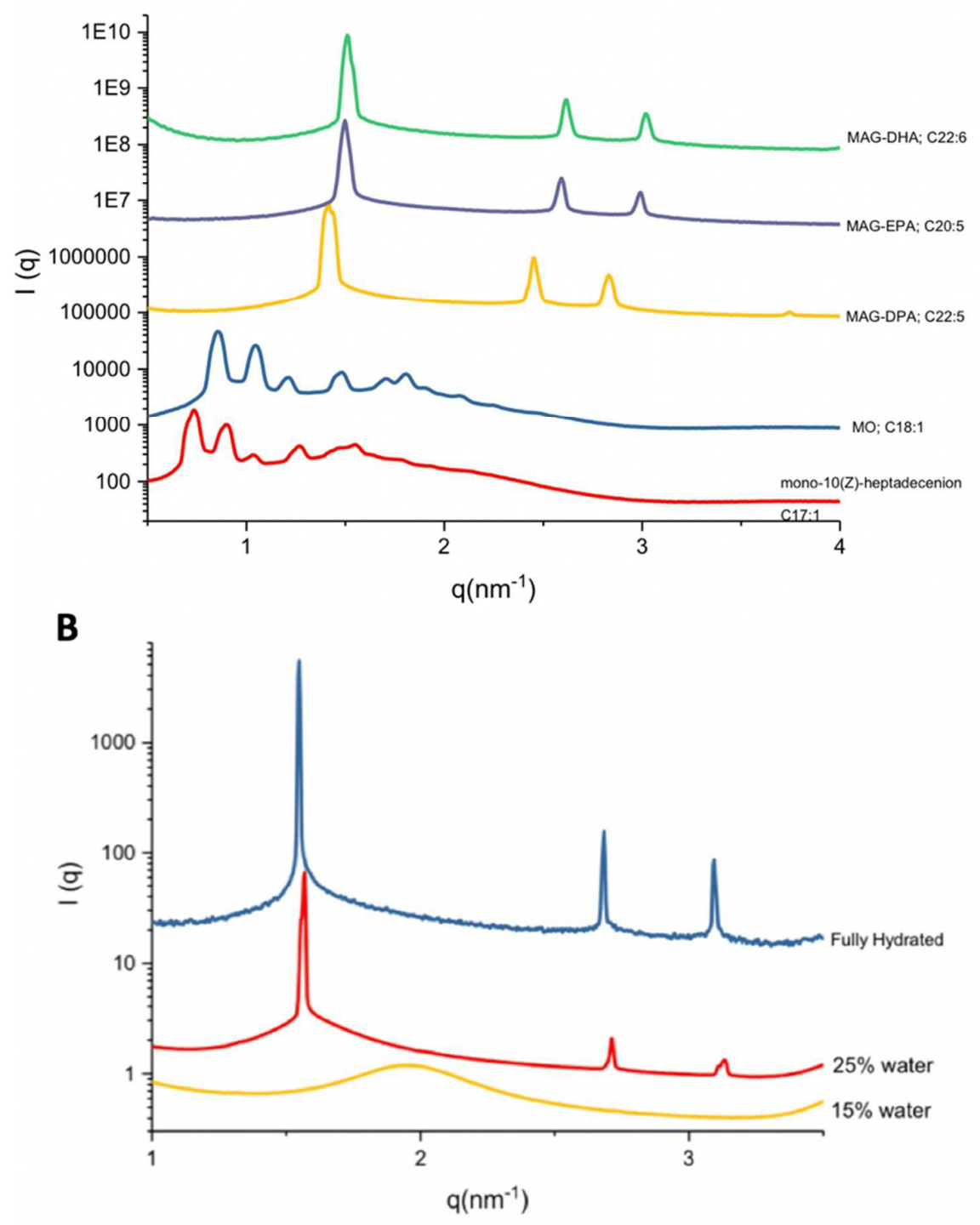


\section{Figure 3}
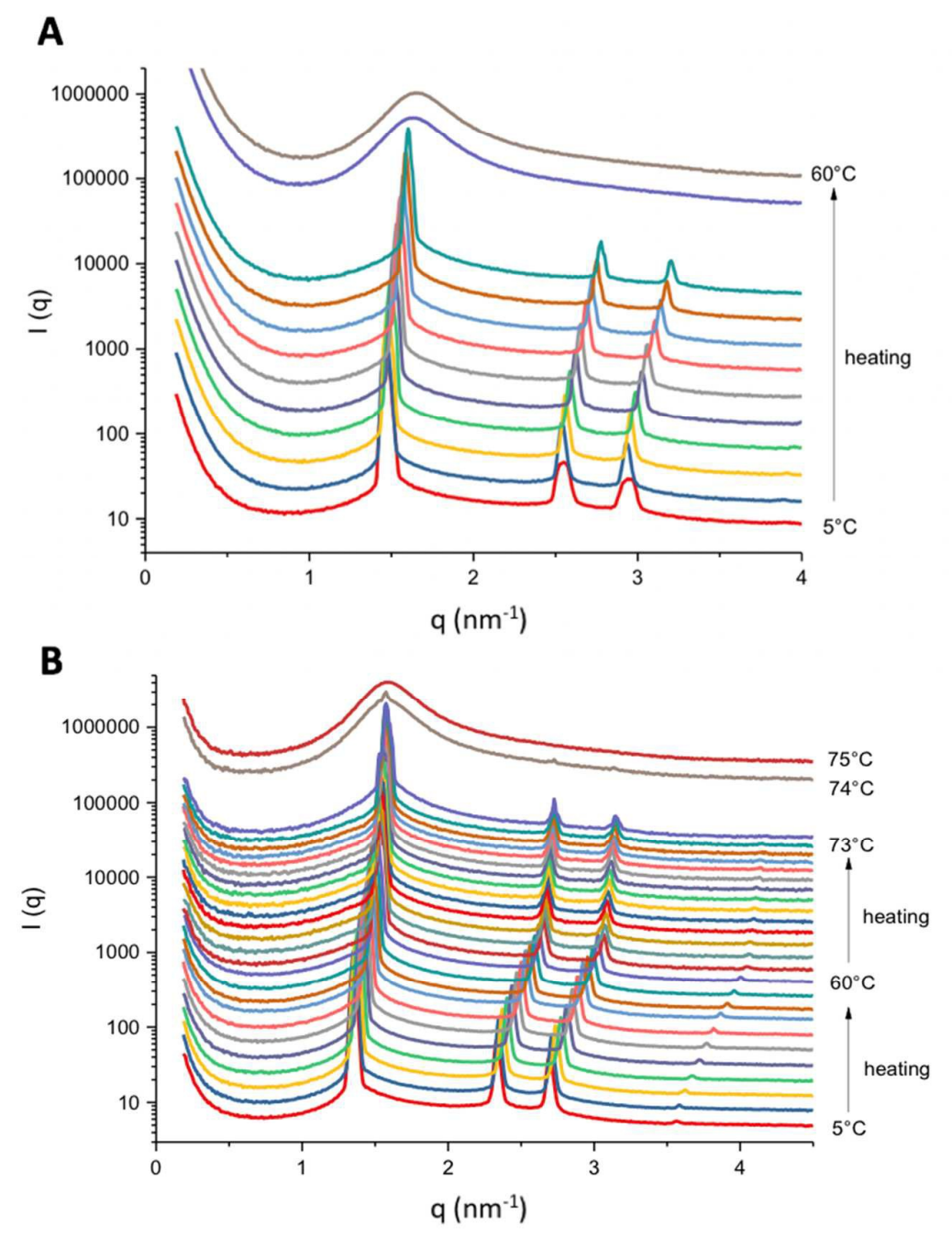


\section{Figure 4}

A

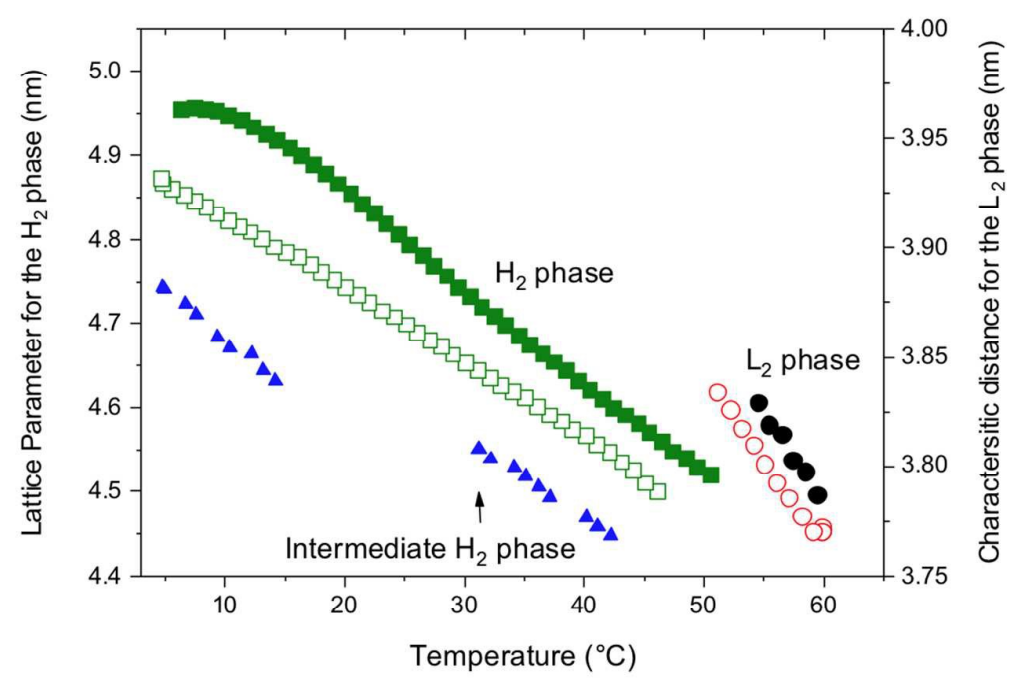

B

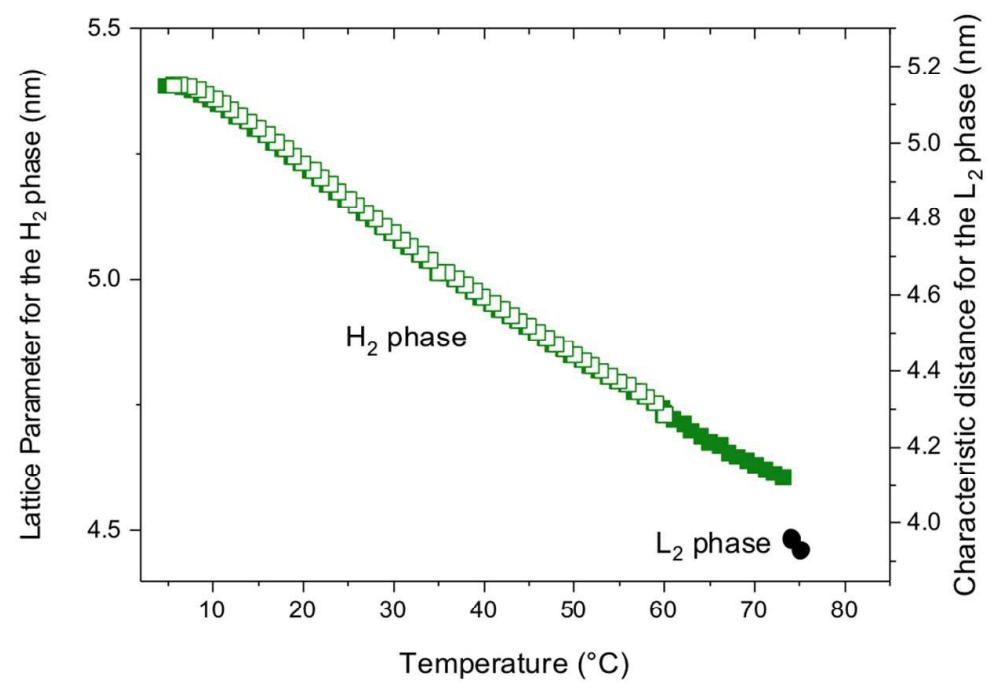




\section{Figure 5}
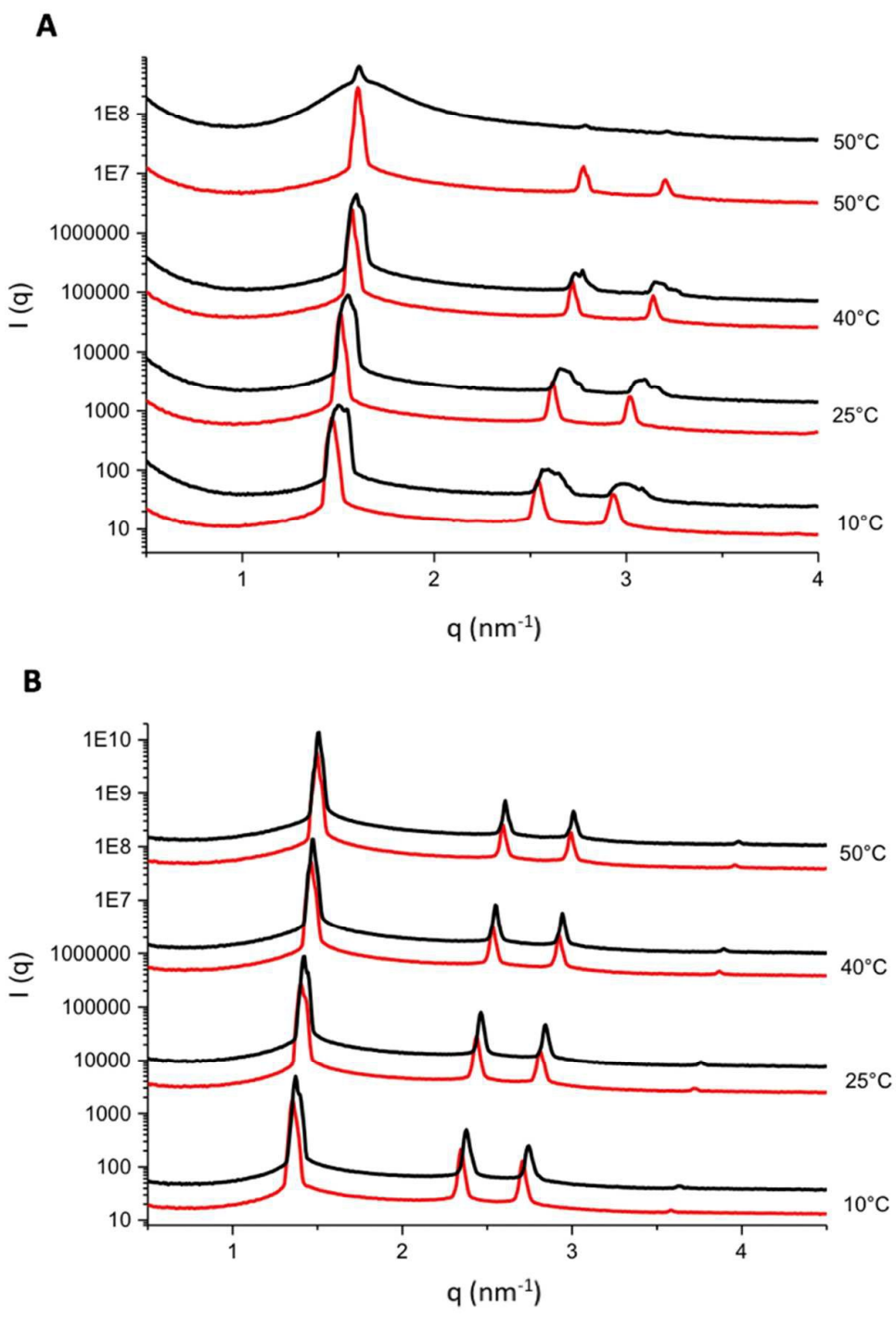


\section{Figure 6}

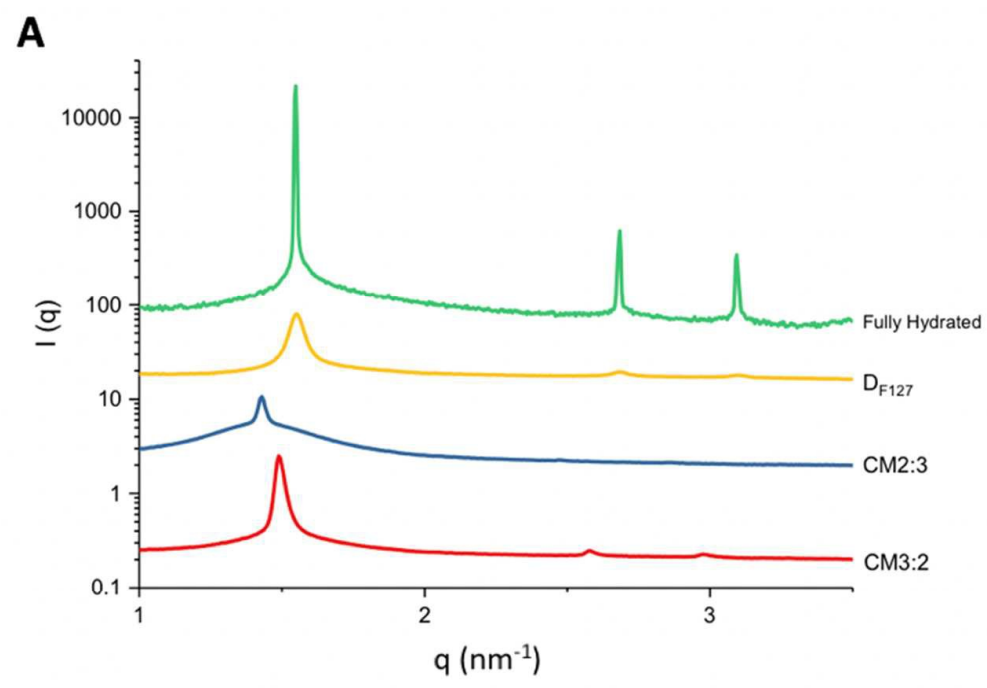

B

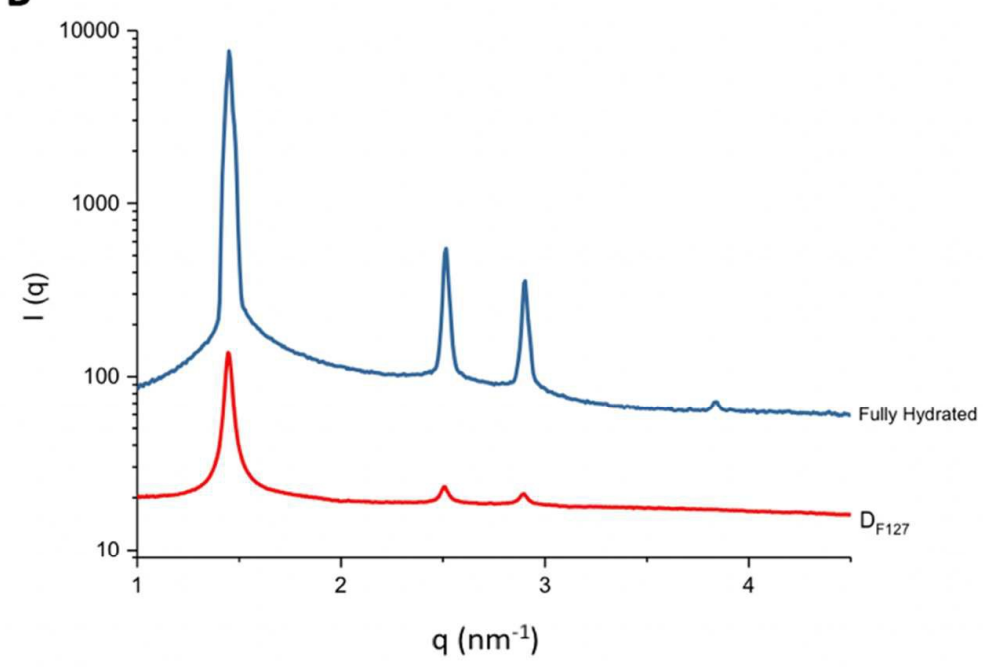




\section{Figure 7}

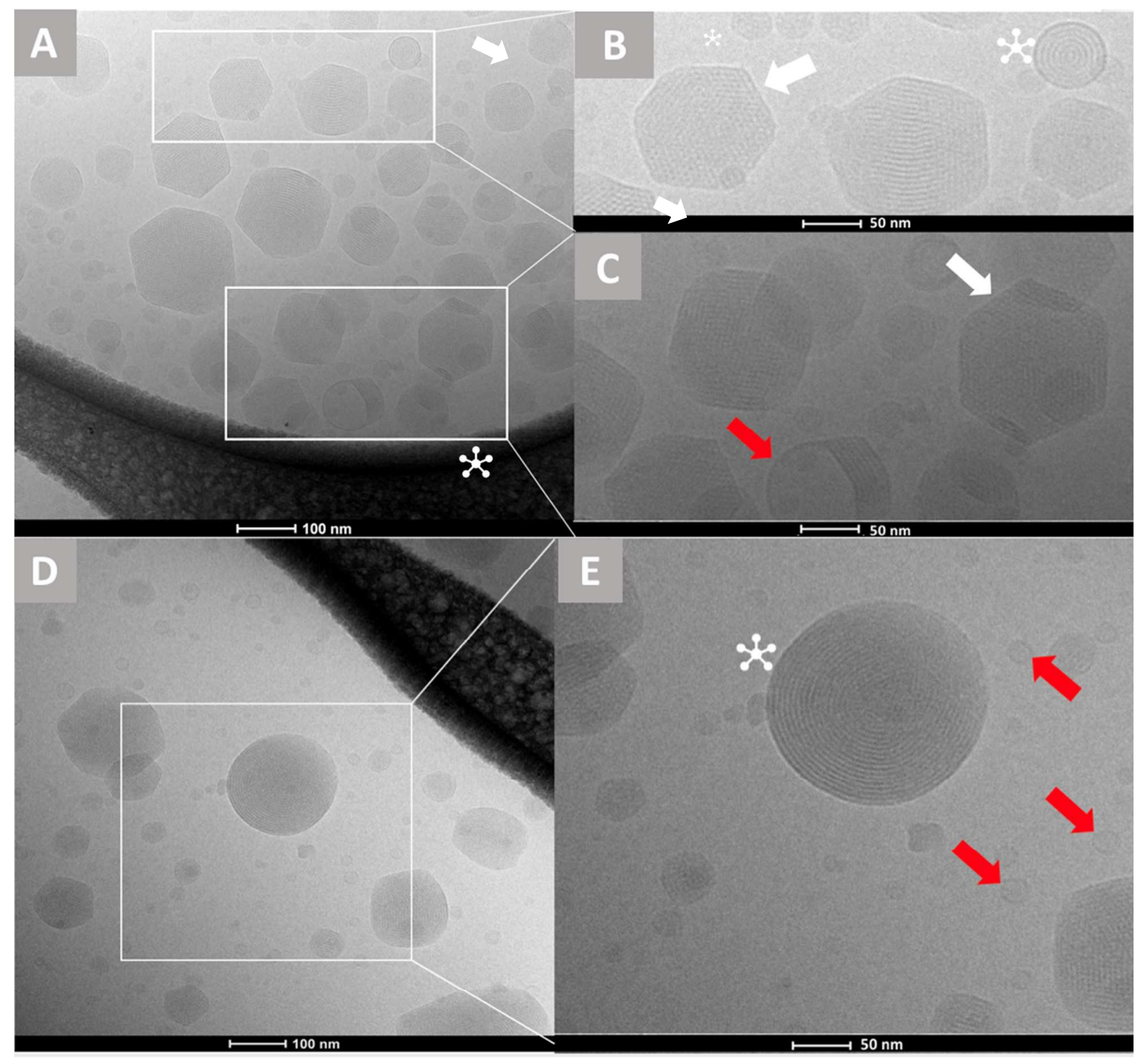




\section{Figure 8}

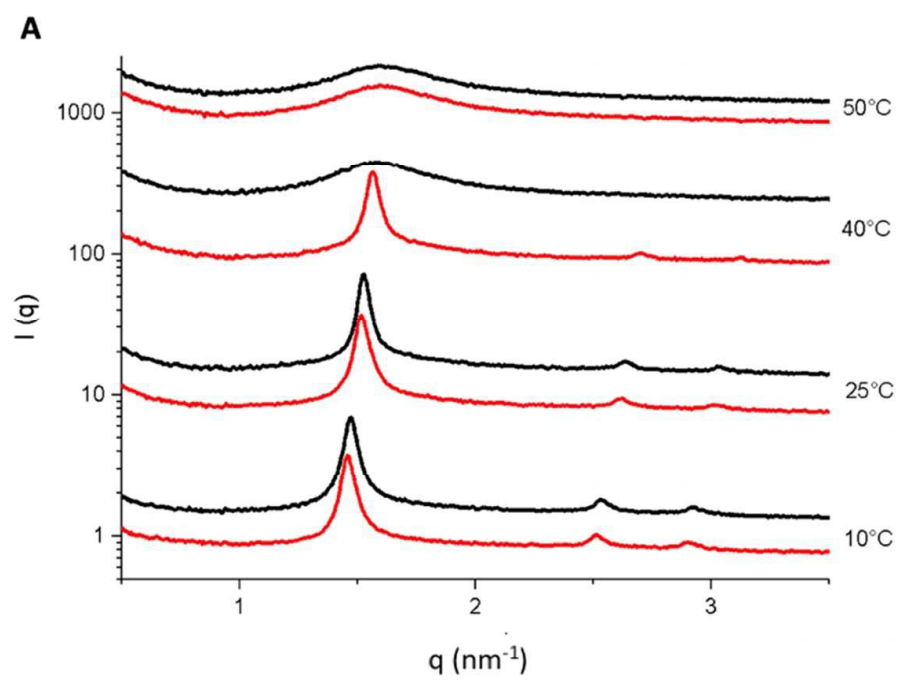

B

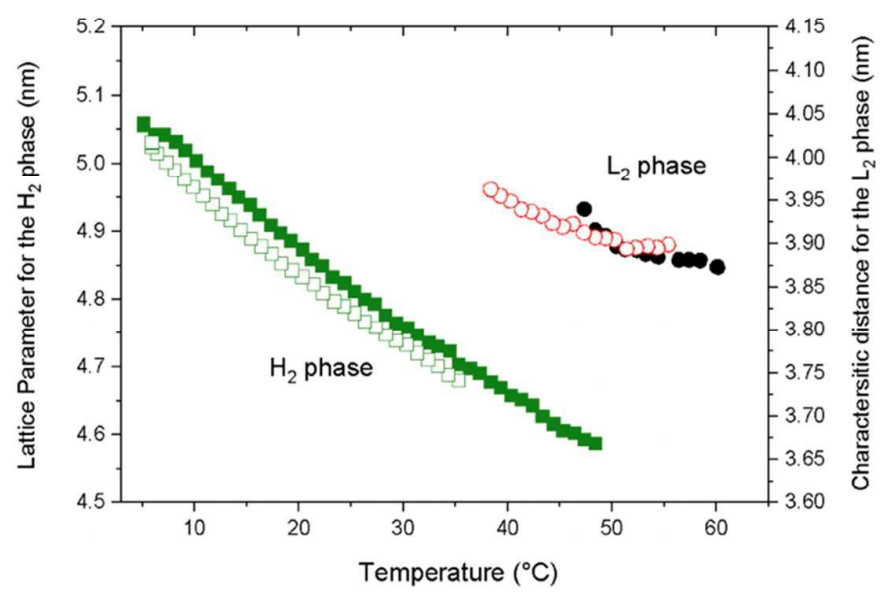




\section{Figure 9}
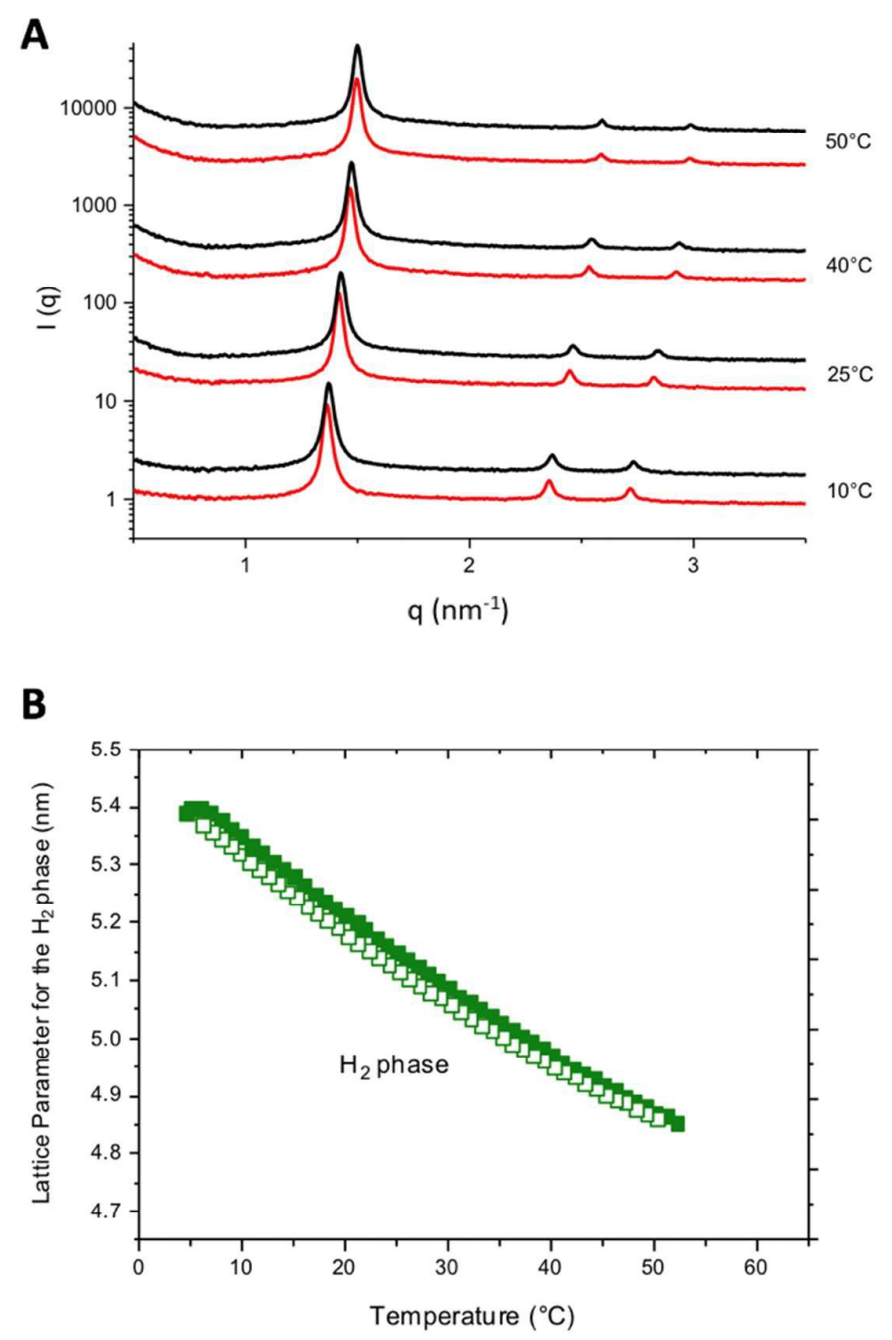


\section{Figure 10}
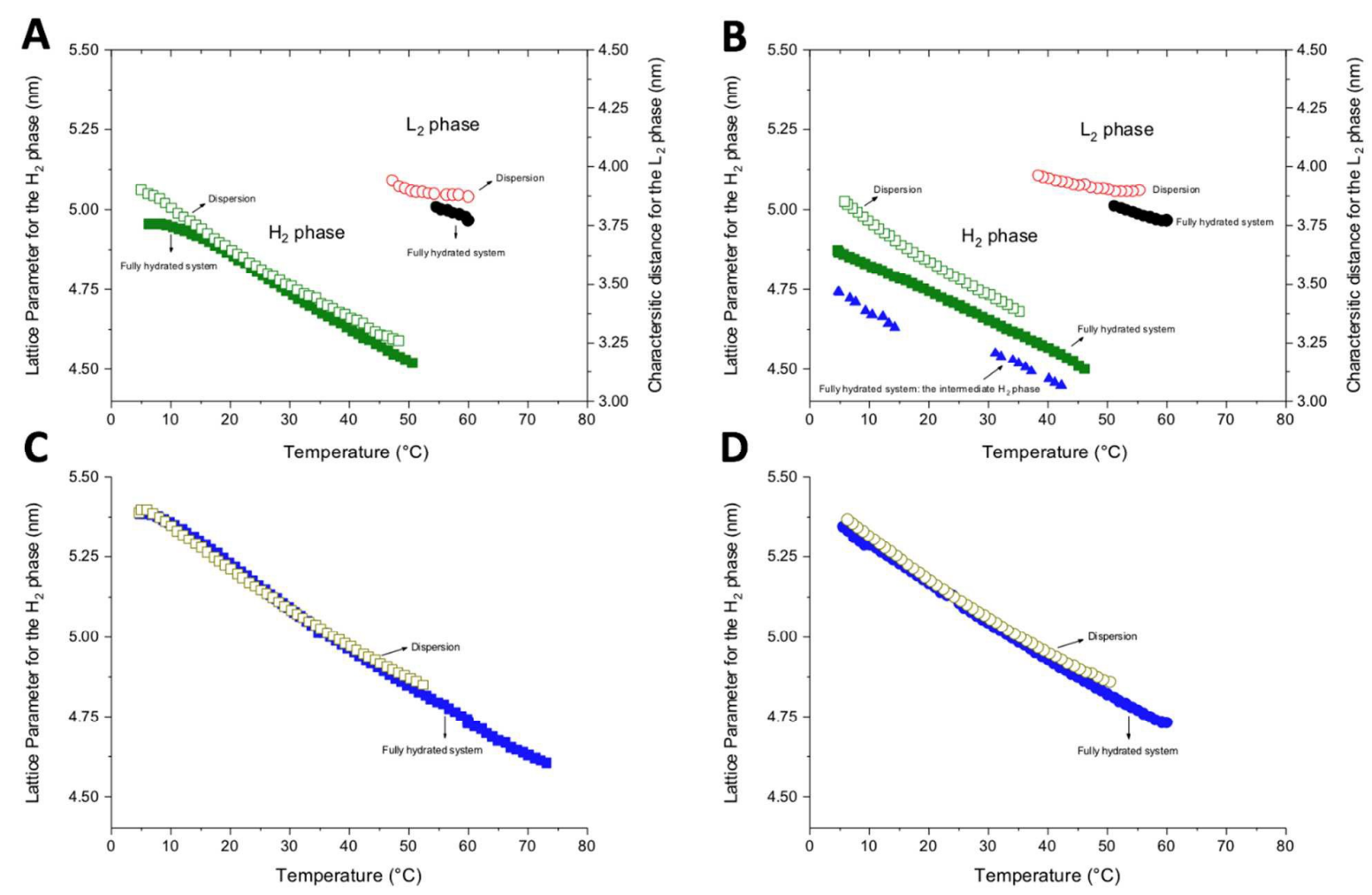


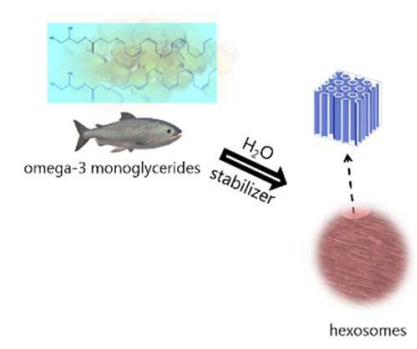

Hexosomes based on omega-3 polyunsaturated fatty acid Monoglycerides 